\title{
POWER-LAW BEHAVIOUR, HETEROGENEITY, AND TREND CHASING
}

XUE-ZHONG HE* AND YOUWEI LI**

\author{
*School of Finance and Economics \\ University of Technology, Sydney \\ PO Box 123 Broadway \\ NSW 2007, Australia \\ and \\ **School of Management and Economics \\ Queen's University, Belfast \\ 25 University Square \\ BT7 1NN, Belfast, UK
}

Key words and phrases. Asset pricing, fundamentalists and trend followers, market fraction, stability, learning, power-law.

*Acknowledgements: This research was initiated during a sabbatical visit of He at the University of Amsterdam and Kiel University. We are indebted to Carl Chiarella, Bas Donkers, Cars Hommes, Thomas Lux and Bertrand Melenberg for many stimulating discussions. The authors would like to thank the referees for their insightful reports and many helpful suggestions. The usual caveat applies. Financial support from the Australian Research Council (ARC) under discovery grant (DP0450526), and the University of Technology, Sydney under a research excellence grant are also gratefully acknowledged.

Corresponding author: Xuezhong (Tony) He, School of Finance and Economics, University of Technology, Sydney, PO Box 123 Broadway, NSW 2007, Australia. Email: Tony.He1@uts.edu.au. Ph: (61 2) 9514 7726. Fax: (61 2) 95147722 . 


\begin{abstract}
Long-range dependence in volatility is one of the most prominent examples in financial market research involving universal power laws. Its characterization has recently spurred attempts to provide some explanations of the underlying mechanism. This paper contributes to this recent line of research by analyzing a simple market fraction asset pricing model with two types of traders-fundamentalists who trade on the price deviation from estimated fundamental value and trend followers whose conditional mean and variance of the trend are updated through a geometric learning process. Our analysis shows that agent heterogeneity, risk-adjusted trend chasing through the geometric learning process, and the interplay of noisy fundamental and demand processes and the underlying deterministic dynamics can be the source of power-law distributed fluctuations. In particular, the noisy demand plays an important role in the generation of insignificant autocorrelations (ACs) on returns, while the significant decaying AC patterns of the absolute returns and squared returns are more influenced by the noisy fundamental process. A statistical analysis based on Monte Carlo simulations is conducted to characterize the decay rate. Realistic estimates of the power-law decay indices and the (FI)GARCH parameters are presented.
\end{abstract}

JEL Classification: C15, D84, G12 


\section{INTRODUCTION}

It is well known that (high-frequency) financial time series share some common features, the so called stylized facts ${ }^{1}$; including excess volatility (relative to the dividends and underlying cash flows), volatility clustering (high/low fluctuations are followed by high/low fluctuations), skewness, and excess kurtosis. Traditional economic and finance theory based on the representative agent with rational expectations has encountered great difficulties in explaining these facts. As a result there has been an increase in interest in models incorporating heterogeneous agents and bounded rationality. These models characterize the dynamics of financial asset prices resulting from the interaction of heterogeneous agents having different attitudes to risk and having different expectations about the future evolution of prices ${ }^{2}$. Some of these models derive their price dynamics from nonlinear trading rules while others consider some nonlinear switching mechanism between different trading strategies.

One of the key aspects of these models is that they exhibit feedback of expectations-the agents' decisions are based upon predictions of future values of endogenous variables whose actual values are determined by equilibrium equations. In particular, Brock and Hommes (1997, 1998) proposed an Adaptive Belief System model of economic and financial markets. The agents adapt their beliefs over time by choosing from different predictors or expectations functions, based upon their past performance. The resulting nonlinear dynamical system is, as Brock and Hommes (1998) and Hommes (2002) show, capable of generating a wide range of complex behaviour from local stability to high order cycles and chaos. They are also capable of explaining some of the stylized facts of financial markets. It is very interesting to find that adaptation, evolution, heterogeneity, and even learning, can be incorporated into the Brock and Hommes type of framework. This broader framework also gives rise to rich and complicated dynamics and can be used to obtain a deeper understanding of market behaviour ${ }^{3}$. Moreover, recent works by Westerhoff (2004), Chiarella et al. (2005, 2006a) and Westerhoff and Dieci (2006) show that complex price dynamics may also result within a multi-asset market framework.

\footnotetext{
${ }^{1}$ See Pagan (1996) for a comprehensive discussion of stylized facts characterizing financial time series. ${ }^{2}$ For a representative sample of this literature see, Frankel and Froot (1987), Day and Huang (1990), De Long et al. (1990), Chiarella (1992), Dacorogna et al. (1995), Lux (1995, 1997, 1998), Brock and LeBaron (1996), Arthur et al. (1997), Brock and Hommes (1997, 1998), Chen and Yeh (1997, 2002), Lux and Marchesi (1999), Bullard and Duffy (1999), LeBaron et al. (1999), LeBaron (2000, 2001, 2002), Iori (2002), Hommes (2002) and Farmer and Joshi (2002).

${ }^{3}$ In this regard see, Gaunersdorfer (2000), Hommes (2001, 2002), Chiarella and He (2001, 2002, 2003), Chiarella et al. (2002), De Grauwe and Grimaldi (2003) and Westerhoff (2003).
} 
Among the stylized facts, volatility clustering and long-range dependence (that is, insignificant autocorrelations (ACs) of raw returns and hyperbolic decline of ACs of the absolute and squared returns) have been extensively studied since the seminal paper of Ding et al. (1993). Recently, a number of universal power laws ${ }^{4}$ have been found to hold in financial markets. This finding has spurred attempts at a theoretical explanation and the search for an understanding of the underlying mechanisms responsible for such power laws. ${ }^{5}$ This paper contributes to the development of this literature.

Various models have been developed to explain the power-law behaviour. For instance the popular GARCH class processes, initiated in Engle (1982), model returns as a random process with a time-varying variance that shows autoregressive dependence. These models produce fat tails of the unconditional distribution and capture the short-run dynamics of volatility autocorrelations. However, the implied decay of the volatility autocorrelation of these models is exponential rather than the hyperbolic as observed in high frequency (e.g. daily) data. In addition, the GARCH class of models does not provide an explanation of the empirical regularities referred to earlier.

As a consequence of development in the rational bubble models literature, multiplicative stochastic processes (with multiplicative and additive stochastic components) have been used to explain the power-law behaviour (see Kesten (1973) and Lux (2004)). The power-law exponent can be determined from the distribution of the multiplicative component, not the additive noise components. However, as shown by Lux and Sornette (2002), the range of the exponent required for the rational bubble models is very different from the empirical findings. In addition, rational bubble models share the conceptual problems of economic models with fully rational agents.

Herding models of financial markets have been developed to incorporate herding and contagion phenomena. ${ }^{6}$ Using a stripped down version of an extremely parsimonious stochastic herding model with fundamentalists (who trade on observed mispricing) and noise traders (who follow the mood of the market), Alfarano et al. (2005) show that price changes are generated by either exogenous inflow of new information about fundamentals or endogenous changes in

\footnotetext{
${ }^{4}$ They include cubic power distribution of large returns, hyperbolic decline of the return autocorrelation function, temporal scaling of trading volume and multi-scaling of higher moments of returns.

${ }^{5}$ We refer to Lux (2004) for a recent survey on empirical evidence, models and mechanisms of various financial power laws.

${ }^{6}$ See Kirman (1991, 1993), Lux (1995, 1997, 1998), Lux and Marchesi (1999), Chen et al. (2001), Aoki and Yoshikawa (2002), and Alfarano et al. (2005).
} 
demand and supply via the herding mechanism. The model is able to produce relatively realistic time series for returns whose distributional and temporal characteristics are astonishingly close to the empirical findings. This is partly due to a bi-modal limiting distribution for the fraction of noise traders in the optimistic and pessimistic groups of individuals and partly due to the stochastic nature of the process leading to recurrent switches from one majority to another. Lux and Marchesi (1999) argue that the indeterminateness of the market fractions in a market equilibrium and the dependence of stability on the market fractions exist in a broad class of behaviour al finance models. This argument is supported by Giardina and Bouchaud (2003) and Lux and Schornstein (2005). However, with the increase of the population size, the law of large numbers comes into effect and the indeterminacy and power-law statistics disappear.

As discussed earlier, the Brock and Hommes' framework and its various extensions are capable of explaining various types of market behaviour and important stylized facts. For example, a mechanism of switching between predictors and co-existing attractors is used in Gaunersdorfer and Hommes (2006) to characterize volatility clustering. The highly nonlinear deterministic system may exhibit co-existence of different types of attractors and adding noise to the deterministic system may then trigger switches between low- and high-volatility phases. Their numerical simulations show quite satisfactory statistics between the simulated and actual data. Compared to the herding mechanism, Brock and Hommes' framework allows an infinite population of speculators. However, like most of the analytical heterogeneous agent literature developed so far, the comparison with empirical facts is mainly based upon visual inspection, or upon a few realizations of the model. A formal investigation of the time series properties of the heterogeneous agent models, including the estimation of power-law indices, is still lacking. This paper seeks to fill this gap in the literature.

Overall both the herding and switching models discussed above have shown their potential to explain power-law behaviour. ${ }^{7}$ To generate realistic time series, some kind of intermittent dynamics and self-amplification of fluctuations via herding or technical trading are necessary. As pointed out by Lux (2004), "one of the more important problems of these models is the relationship between system size, deterministic forces and stochastic elements".

\footnotetext{
${ }^{7}$ Other behaviour al finance explanations for volatility clustering exist. Manzan and Westerhoff (2005) develop a model in which traders tend to over or under-react to the arrival of new information.
} 
In this paper, we consider the market fraction (MF) model established in He and Li (2005) and explore the potential mechanism of the model to generate the power-law feature observed in empirical data. The MF model is a simple stochastic asset pricing model, involving two types of traders (fundamentalists and trend followers) under a market maker scenario. $\mathrm{He}$ and $\mathrm{Li}$ (2005) aim to explain various aspects of financial market behaviour and establish the connection between the stochastic model and its underlying deterministic system. Through a statistical analysis, the paper shows that convergence of market price to fundamental value, long- and short-run profitability of the two trading strategies, survivability of trend followers and various under- and over-reaction autocorrelation patterns of the stochastic model can be explained by the dynamics, including the stability and bifurcations, of the underlying deterministic system.

This paper builds on He and Li (2005) and reveals the potential of the MF model to characterize the volatility clustering and the long-range dependence of asset returns. We show that heterogeneity, risk-adjusted trend chasing through a geometric learning process, and the interplay of a stable deterministic equilibrium and stochastic noisy processes can be the source of power-law distributed fluctuations. This is further verified via a Monte Carlo simulation, a statistical analysis of the decay patterns of autocorrelation functions of returns, the squared returns and the absolute returns, and the estimates of $(\mathrm{FI}) \mathrm{GARCH}(1,1)$ parameters. Both the analysis of the generating mechanism and the statistical estimates via a Monte Carlo simulation of the power-law behaviour are the main contributions of the current paper.

The remainder of the paper is organized as follows. Section 2 reviews the MF model established in He and Li (2005). Section 3 is devoted to an analysis of the potential of the MF model to generate the power-law behaviour. In Section 4 we estimate the power-law decay parameters of the autocorrelation of returns, the squared returns and the absolute returns and (FI)GARCH(1,1) parameters for the DAX 30, the FTSE 100, the NIKKEI 225 and the S\&P 500 stock market daily closing price indices. The power-law properties of the market fraction model and the comparison with the actual data is analyzed in Section 5. Section 6 concludes.

\section{The Market Fraction Model}

The market fraction (MF) model is a standard discounted value asset pricing model with heterogeneous agents. It is closely related to the framework of Brock and Hommes (1997, 
1998) and Chiarella and He (2002). Here we outline the model and refer the readers to He and Li (2005) for full details.

Consider an economy with one risky asset and one risk free asset. It is assumed that the risk free asset is perfectly elastically supplied at gross return of $R=1+r / K$, where $r$ stands for a constant risk-free rate per annum and $K$ stands for the trading frequency measured in units of a year. ${ }^{8}$ Let $P_{t}$ and $D_{t}$ be the (ex dividend) price and dividend per share of the risky asset at time $t$, respectively. Then the wealth of a typical investor- $h$ at $t+1, W_{h, t+1}$, is given by

$$
W_{h, t+1}=R W_{h, t}+\left[P_{t+1}+D_{t+1}-R P_{t}\right] z_{h, t}
$$

where $z_{h, t}$ is the number of shares of the risky asset purchased by investor- $h$ at $t$. Let $E_{h, t}$ and $V_{h, t}$ be the beliefs of type $h$ traders about the conditional expectation and variance at $t+1$ based on their information at time $t$. Denote by $R_{t+1}\left(=P_{t+1}+D_{t+1}-R P_{t}\right)$ the excess capital gain on the risky asset at $t+1$. Assume that type $h$ traders have constant absolute risk aversion (CARA) utility functions with the risk aversion coefficient $a_{h}$ (that is $U_{h}(W)=-e^{-a_{h} W}$ ) and their optimal demands for the risky asset $z_{h, t}$ are determined by maximizing their expected utility of wealth. Then it turns out that

$$
z_{h, t}=\frac{E_{h, t}\left(R_{t+1}\right)}{a_{h} V_{h, t}\left(R_{t+1}\right)}
$$

Given the heterogeneity and the nature of asymmetric information among traders, we consider two popular trading strategies corresponding to two types of boundedly rational tradersfundamentalists and trend followers. Assume that the market fractions of the fundamentalists and trend followers are $n_{1}$ and $n_{2}$, respectively. Let $m=n_{1}-n_{2} \in[-1,1]$, then $m=1(-1)$ corresponds to the case when all the traders are fundamentalists (trend followers). Assume zero supply of outside shares. Then, using (2.2), the population weighted aggregate excess demand $z_{e, t}$ is given by

$$
z_{e, t} \equiv n_{1} z_{1, t}+n_{2} z_{2, t}=\frac{1+m}{2} \frac{E_{1, t}\left[R_{t+1}\right]}{a_{1} V_{1, t}\left[R_{t+1}\right]}+\frac{1-m}{2} \frac{E_{2, t}\left[R_{t+1}\right]}{a_{2} V_{2, t}\left[R_{t+1}\right]} .
$$

\footnotetext{
${ }^{8}$ Typically, $K=1,12,52$ and 250 representing trading periods of year, month, week and day, respectively. To calibrate the stylized facts observed from daily price movement in financial market, we select $K=250$ in our discussion.
} 
To complete the model, we assume that the market is cleared by a market maker. The role of the market maker is to take a long (when $z_{e, t}<0$ ) or short (when $z_{e, t}>0$ ) position so as to clear the market. At the end of period $t$, after the market maker has carried out all transactions, he or she adjusts the price for the next period in the direction of the observed excess demand. Let $\mu$ be the speed of price adjustment of the market maker (this can also be interpreted as the market aggregate risk tolerance). To capture unexpected market news or the excess demand of noise traders, we introduce a noisy demand term $\tilde{\delta}_{t}$ which is an i.i.d. normally distributed random variable with $\tilde{\delta}_{t} \sim \mathcal{N}\left(0, \sigma_{\delta}^{2}\right)$. Based on these assumptions and (2.3), the market price is determined by

$$
P_{t+1}=P_{t}+\frac{\mu}{2}\left[(1+m) \frac{E_{1, t}\left[R_{t+1}\right]}{a_{1} V_{1, t}\left[R_{t+1}\right]}+(1-m) \frac{E_{2, t}\left[R_{t+1}\right]}{a_{1} V_{2, t}\left[R_{t+1}\right]}\right]+\tilde{\delta}_{t}
$$

Now we turn to discuss the beliefs of fundamentalists and trend followers.

Fundamentalists-Denote by $F_{t}=\left\{P_{t}, P_{t-1}, \cdots ; D_{t}, D_{t-1}, \cdots\right\}$ the common information set formed at time $t$. Apart from the common information set, the fundamentalists are assumed to have superior information on the fundamental value, $P_{t}^{*}$, of the risky asset which is introduced as an exogenous news arrival process. More precisely, the relative return $\left(P_{t+1}^{*} / P_{t}^{*}-1\right)$ of the fundamental value is assumed to follow a normal distribution, and hence we write

$$
P_{t+1}^{*}=P_{t}^{*}\left[1+\sigma_{\epsilon} \tilde{\epsilon}_{t}\right], \quad \tilde{\epsilon}_{t} \sim \mathcal{N}(0,1), \quad \sigma_{\epsilon} \geq 0, \quad P_{0}^{*}=\bar{P}>0,
$$

where $\tilde{\epsilon}_{t}$ is independent of the noisy demand process $\tilde{\delta}_{t}$. This specification ensures that neither fat tails nor volatility clustering are brought about by the exogenous news arrival process. Hence, emergence of any autocorrelation pattern of the return of the risky asset in our later discussion would be driven by the trading process itself, rather than news. The fundamentalists also realize the existence of non-fundamental traders, such as trend followers to be introduced in the following discussion. The fundamentalists believe that the stock price may be driven away from the fundamental value in the short-run, but it will eventually converge to the expected fundamental value in the long-run. Hence the conditional mean and variance of the fundamental traders are assumed to follow

$$
E_{1, t}\left(P_{t+1}\right)=P_{t}+\alpha\left[E_{1, t}\left(P_{t+1}^{*}\right)-P_{t}\right], \quad V_{1, t}\left(P_{t+1}\right)=\sigma_{1}^{2},
$$


where $\sigma_{1}^{2}$ stands for a constant variance of the fundamental value. Here the parameter $\alpha \in$ $[0,1]$ represents the speed of price adjustment of the fundamentalists toward their expected fundamental value and it measures how fast the fundamentalists believe the price converges to the fundamental value and reflects how confident they are in the fundamental value. In particular, for $\alpha=1$, the fundamental traders are fully confident about the fundamental value and adjust their expected price in the next period instantaneously to the expected fundamental value. For $\alpha=0$, the fundamentalists become naive traders.

Trend followers - Unlike the fundamental traders, trend followers are technical traders who believe the future price change can be predicted from various patterns or trends generated from the historical prices. They are assumed to extrapolate the latest observed price change over a long-run sample mean price and to adjust their variance estimate accordingly. More precisely, their conditional mean and variance are assumed to satisfy

$$
E_{2, t}\left(P_{t+1}\right)=P_{t}+\gamma\left(P_{t}-u_{t}\right), \quad V_{2, t}\left(P_{t+1}\right)=\sigma_{1}^{2}+b_{2} v_{t}
$$

where $\gamma, b_{2} \geq 0$ are constants, and $u_{t}$ and $v_{t}$ are the sample mean and variance, respectively, which may be generated from some learning processes. The parameter $\gamma$ measures the extrapolation rate and high (low) values of $\gamma$ correspond to strong (weak) extrapolation by the trend followers. The coefficient $b_{2}$ measures the influence of the sample variance on the conditional variance estimated by the trend followers who believe in more volatile price movements. Intuitively, the trend followers reduce their demand for the risky asset when the estimated risk is high. It turns out that this risk-adjusted demand mechanism plays a very important role in the price dynamics ${ }^{9}$. Various learning schemes (see for example Chiarella and He $(2002,2003)$ ) can be used to estimate the sample mean $u_{t}$ and variance $v_{t}$. Here we assume that

$$
u_{t}=\delta u_{t-1}+(1-\delta) P_{t}, \quad v_{t}=\delta v_{t-1}+\delta(1-\delta)\left(P_{t}-u_{t-1}\right)^{2}
$$

where $\delta \in[0,1]$ is a constant. These processes for the sample mean and variance are the limit of a geometric decay process when the memory lag length tends to infinity ${ }^{10}$. Basically, a geometric decay probability process $(1-\delta)\left\{1, \delta, \delta^{2}, \cdots\right\}$ is associated with the historical prices

\footnotetext{
${ }^{9} \mathrm{~A}$ similar set up under a different learning process is used in Chiarella et al. (2006c) who show that the timevarying second moment can alter the resulting nonlinear dynamics, particularly when the steady state is unstable.

${ }^{10}$ See Chiarella et. al. (2006b) for the proof.
} 
$\left\{P_{t}, P_{t-1}, P_{t-2}, \cdots\right\}$. The parameter $\delta$ measures the geometric decay rate ${ }^{11}$. The rationale for the selection of this process is two fold. First, traders tend to put a high weight on the most recent prices and less weight on the more remote prices when they estimate the sample mean and variance. Secondly, we believe that this geometric decay process may contribute to certain autocorrelation patterns, in particular the power-law feature observed in real financial markets. In addition, the geometric decay process has the mathematical advantage of affording a degree of tractability to the subsequent analysis.

To simplify the calculations, we assume that the dividend process $D_{t}$ follows $D_{t} \sim \mathcal{N}\left(\bar{D}, \sigma_{D}^{2}\right)$, the expected long-run fundamental value is given by $\bar{P}=\bar{D} /(R-1)$, and the unconditional variances of the price $\left(\sigma_{1}^{2}\right)$ and dividend $\left(\sigma_{D}^{2}\right)$ over the trading period are related ${ }^{12}$ by $\sigma_{D}^{2}=q \sigma_{1}^{2}$. Based on (2.6), we have $E_{1, t}\left(R_{t+1}\right)=\alpha\left(P_{t+1}^{*}-P_{t}\right)-(R-1)\left(P_{t}-\bar{P}\right), V_{1, t}\left(R_{t+1}\right)=(1+q) \sigma_{1}^{2}$ and hence the optimal demand of the fundamentalist is given by

$$
z_{1, t}=\frac{1}{a_{1}(1+q) \sigma_{1}^{2}}\left[\alpha\left(P_{t}^{*}-P_{t}\right)-(R-1)\left(P_{t}-\bar{P}\right)\right]
$$

Similarly, from (2.7), $E_{2, t}\left(R_{t+1}\right)=P_{t}+\gamma\left(P_{t}-u_{t}\right)+\bar{D}-R P_{t}=\gamma\left(P_{t}-u_{t}\right)-(R-1)\left(P_{t}-\right.$ $\bar{P}), V_{2, t}\left(R_{t+1}\right)=\sigma_{1}^{2}\left(1+q+b v_{t}\right)$, where $b=b_{2} / \sigma_{1}^{2}$. Hence the optimal demand of the trend followers is given by

$$
z_{2, t}=\frac{\gamma\left(P_{t}-u_{t}\right)-(R-1)\left(P_{t}-\bar{P}\right)}{a_{2} \sigma_{1}^{2}\left(1+q+b v_{t}\right)}
$$

\footnotetext{
${ }^{11}$ For $\delta=0$, the sample mean $u_{t}=P_{t}$, which is the latest observed price, while $\delta=0.1,0.5,0.95$ and 0.999 give a half lives of 0.43 day, 1 day, 2.5 weeks and 2.7 years, respectively.

${ }^{12}$ Let $\sigma_{\bar{P}}$ be the annual volatility of $P_{t}^{*}$ and $\bar{D}_{t}=r P_{t}^{*}$ be the annual dividend. In this paper, we choose $\sigma_{1}^{2}=$ $\sigma_{\bar{P}}^{2} / K$ and $q=r^{2}$. In fact, the annual variance of the dividend is $\bar{\sigma}_{D}^{2}=r^{2} \sigma_{\bar{P}}^{2}$. Therefore $\sigma_{D}^{2}=\bar{\sigma}_{D}^{2} / K=$ $r^{2} \sigma_{\bar{P}}^{2} / K=r^{2} \sigma_{1}^{2}$. For all numerical simulations in this paper, we choose $\bar{P}=\$ 100, r=5 \%$ p.a. $\sigma=20 \%$ p.a., $\sigma_{\bar{P}}=\sigma \bar{P}$ and $K=250$. Correspondingly, $R=1+0.05 / 250=1.0002, \sigma_{1}^{2}=(100 \times 0.2)^{2} / 250=8 / 5$ and $\sigma_{D}^{2}=1 / 250$.
} 
Subsisting (2.9) and (2.10) into (2.4), the market price under a market maker is determined by the following 4-dimensional stochastic difference system

$$
\left\{\begin{aligned}
& P_{t+1}= P_{t}+\frac{\mu}{2}\left[\frac{1+m}{a_{1}(1+q) \sigma_{1}^{2}}\left[\alpha\left(P_{t}^{*}-P_{t}\right)-(R-1)\left(P_{t}-\bar{P}\right)\right]\right. \\
&\left.+(1-m) \frac{\gamma\left(P_{t}-u_{t}\right)-(R-1)\left(P_{t}-\bar{P}\right)}{a_{2} \sigma_{1}^{2}\left(1+q+b v_{t}\right)}\right]+\tilde{\delta}_{t} \\
& u_{t}= \delta u_{t-1}+(1-\delta) P_{t} \\
& v_{t}=\delta v_{t-1}+\delta(1-\delta)\left(P_{t}-u_{t-1}\right)^{2}
\end{aligned}\right.
$$

By using Monte Carlo simulation and statistical analysis, He and Li (2005) found that the long-run behaviour and convergence of the market prices, long (short)-run profitability of the fundamental (trend following) trading strategy, survivability of trend followers, and various under and over-reaction autocorrelation patterns of returns can be characterized by the dynamics, including the stability and bifurcations, of the underlying deterministic system. The analysis provides some insights into the generating mechanism of various types of market behaviour (such as under/over-reaction), market dominance and stylized facts in high frequency financial markets. In the following discussion, we reveal the potential of the MF model to characterize the volatility clustering and the long-range dependence of asset returns by examining the autocorrelation patterns under different noise structures and by estimating the decay indices and (FI)GARCH parameters.

\section{Analysis of the Volatility Clustering and Power Law Behaviour}

We now proceed with an analysis of the volatility clustering and power-law mechanism of the MF model. The aim of the analysis is to explore possible sources of volatility fluctuations. In doing so, we provide some insights into the interplay between system size, deterministic forces and stochastic elements, in particular, the potential for this interplay to generate realistic time series properties.

Aside from the parameter values of which were given previously, the parameters used for the simulations are given in Table $3.1^{13}$.

\footnotetext{
${ }^{13}$ The return volatility $\sigma_{\epsilon}$ of the fundamental value corresponds to an annual volatility of $20 \%$ (hence $\sigma_{\epsilon}=$ $(20 / \sqrt{K}) \%$ with $K=250)$ and the volatility of the noisy demand $\sigma_{\delta}=1$, which is about $1 \%$ of the average
} 
TABLE 3.1. Parameter settings and initial values

\begin{tabular}{cccccccccccc}
\hline$\alpha$ & $\gamma$ & $a_{1}$ & $a_{2}$ & $\mu$ & $m$ & $\delta$ & $b$ & $\sigma_{\epsilon}$ & $\sigma_{\delta}$ & $P_{0}$ & $P_{0}^{*}$ \\
\hline 0.1 & 0.3 & 0.8 & 0.8 & 2 & 0 & 0.85 & 1 & 0.01265 & 1 & 100 & 100 \\
\hline
\end{tabular}

Following from the stability and bifurcation analysis in He and Li (2005), the constant steady state fundamental price $\bar{P}$ of the underlying deterministic system is locally asymptotically stable for chosen parameter constellations. The intuition behind this selection of parameters comes from the analysis of the return autocorrelation (AC) patterns near the Hopf bifurcation boundary conducted in He and $\mathrm{Li}$ (2005). When the market prices converge to the fundamental values in an oscillating manner, the significant AC patterns of returns are washed out by the noisy market demand process with reasonable volatility. On the other hand, the noisy fundamental process seems necessary to generate more realistic price series. The oscillatory convergence of the underlying deterministic system and the noisy fundamental process lead to volatility clustering-high (low) volatility is more likely followed by high (low) volatility.

To see how the price dynamics, in particular, the AC patterns of returns, are affected by different noise processes, we consider the four cases listed in Table 3.2. Case-00 corresponds to

TABLE 3.2. Four Cases of the noisy effect

\begin{tabular}{|c||c|c|c|c|}
\hline Cases & Case-00 & Case-01 & Case-10 & Case-11 \\
\hline$\left(\sigma_{\delta}, \sigma_{\epsilon}\right)$ & $(0,0)$ & $(0,0.01265)$ & $(1,0)$ & $(1,0.01265)$ \\
\hline
\end{tabular}

the deterministic case. Case-01 (Case-10) corresponds to the case with noisy fundamental price (noisy excess demand) only and both noise processes appear in Case-11.

Fig. 3.1 illustrates the price series for the four cases for a typical simulation. The corresponding return series and their density distributions are given in Fig. 3.2 for the three cases involving noise. Fig. 3.3 shows the ACs of returns, absolute returns and squared returns. For comparison, the same set of noisy demand and fundamental processes is used in Case-11. Each simulation is run for 6,000 time periods and the first 1,000 are dropped to wash out the initial effect of the estimates of densities and ACs of returns and to make the estimates robust.

Both Figs. 3.2 and 3.3 show significantly different impacts of the different noise processes on the volatility. In Case-01, the stochastic fundamental price process is the only noise process.

fundamental price level $\bar{P}=\$ 100$. In addition, simulations (not reported here) show that the results obtained in this paper are robust under slight modifications of these parameters. 

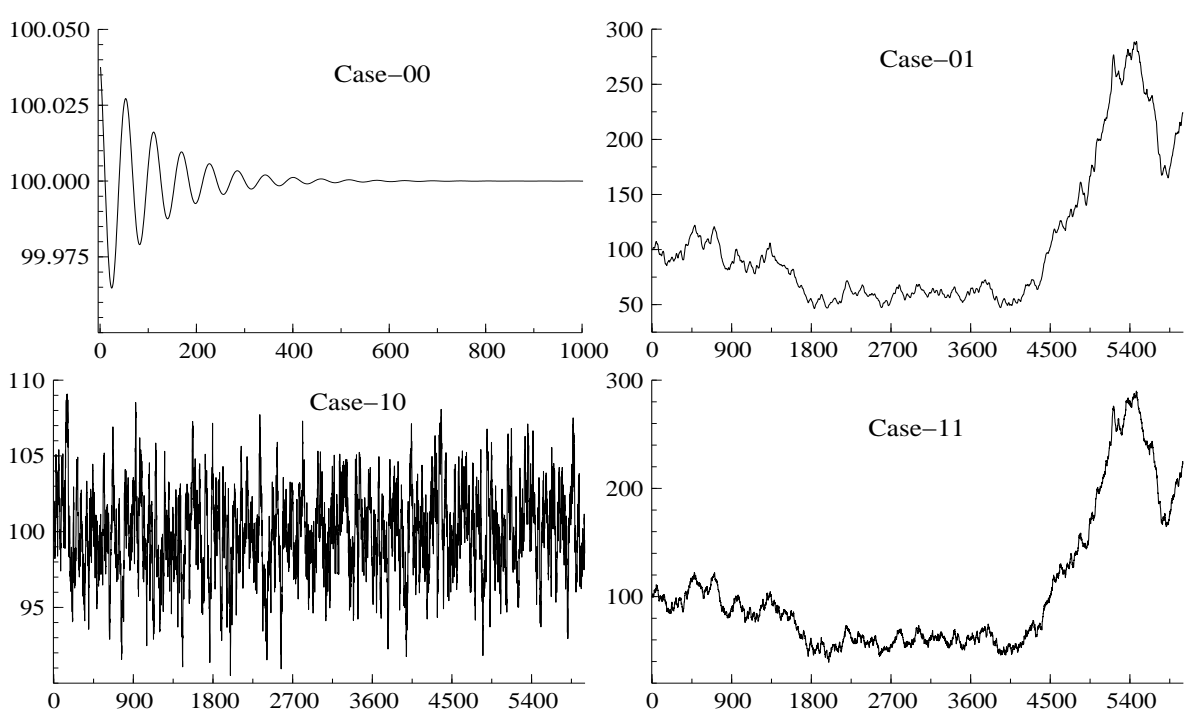

FiguRE 3.1. Time series of prices for the four cases in Table 3.2.
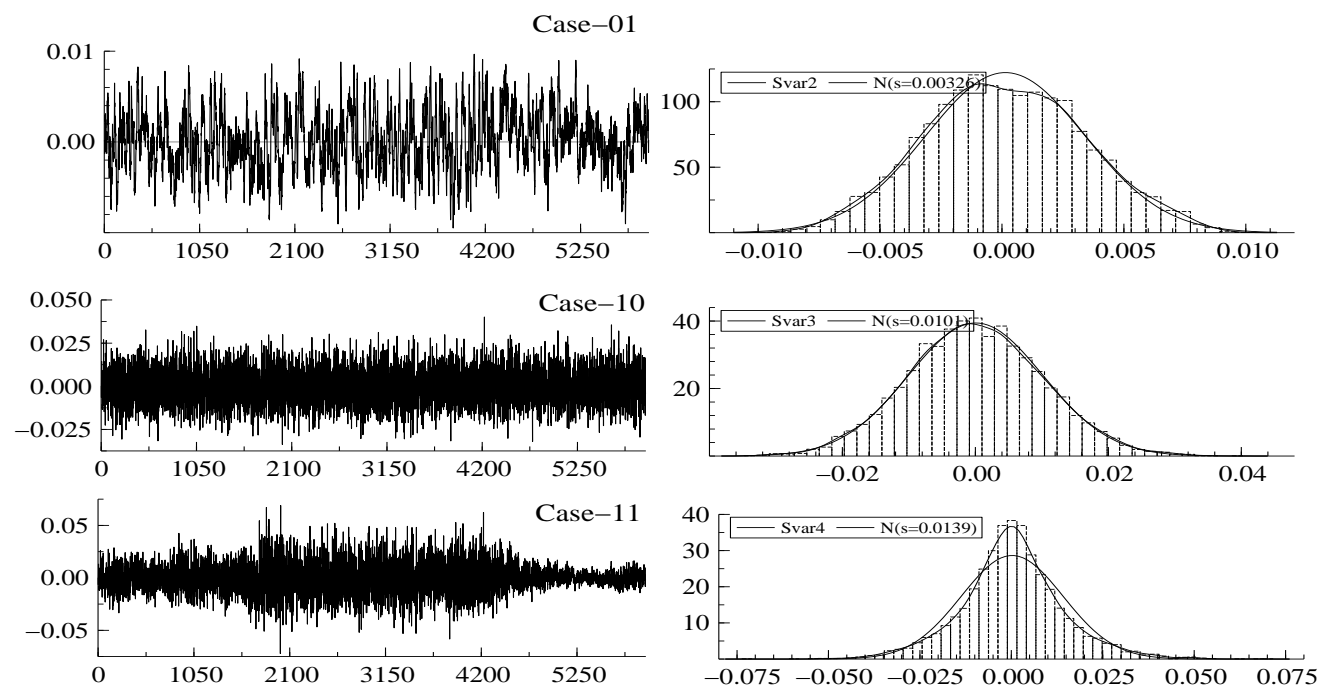

FIGURE 3.2. Return series and their density distributions for Case-01, 10 and 11.

The market price displays a strong under-reaction ${ }^{14}$ AC pattern of returns, which is characterized by the significantly positive decaying ACs shown in the top left panel in Fig. 3.3. This significant AC pattern is also carried forward to the AC patterns for the absolute and squared returns. In Case-10, the noisy excess demand is the only noise process. The market price displays no volatility clustering, which is characterized by insignificant AC patterns for return, the absolute and squared returns shown in the middle row in Fig. 3.3. In Case-11, both the noisy excess demand and noisy fundamental price processes appear. We observe relatively high

\footnotetext{
${ }^{14} \mathrm{See} \mathrm{He}$ and $\mathrm{Li}$ (2005) for more detailed analysis on the generating mechanism for various under- and overreaction AC patterns.
} 

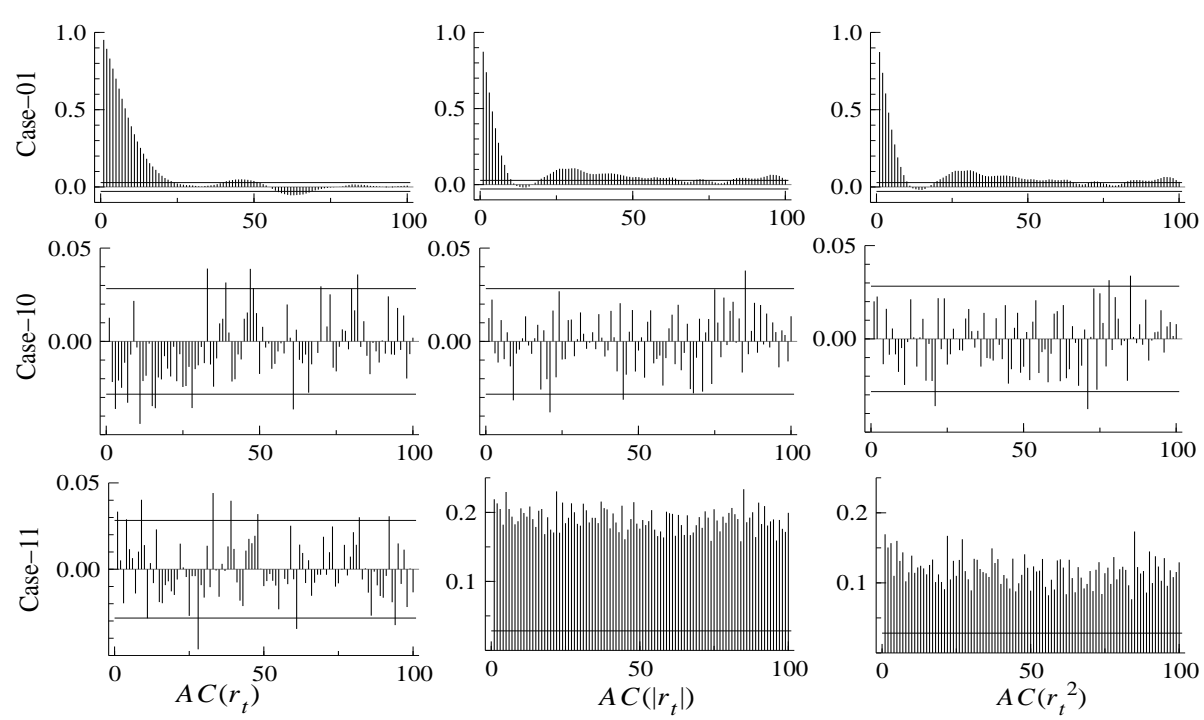

FIGURE 3.3. ACs of returns (the left column), the absolute returns (the middle column), and the squared returns (the right column) for Case-01, 10 and 11.

kurtosis in Fig. 3.2 and insignificant ACs for returns, but significant ACs for the absolute and squared returns shown in the bottom panel in Fig. 3.3. In fact, the estimates in Section 5 based on Monte Carlo simulations show that the model is able to produce relatively realistic volatility pattern and the power-law features.
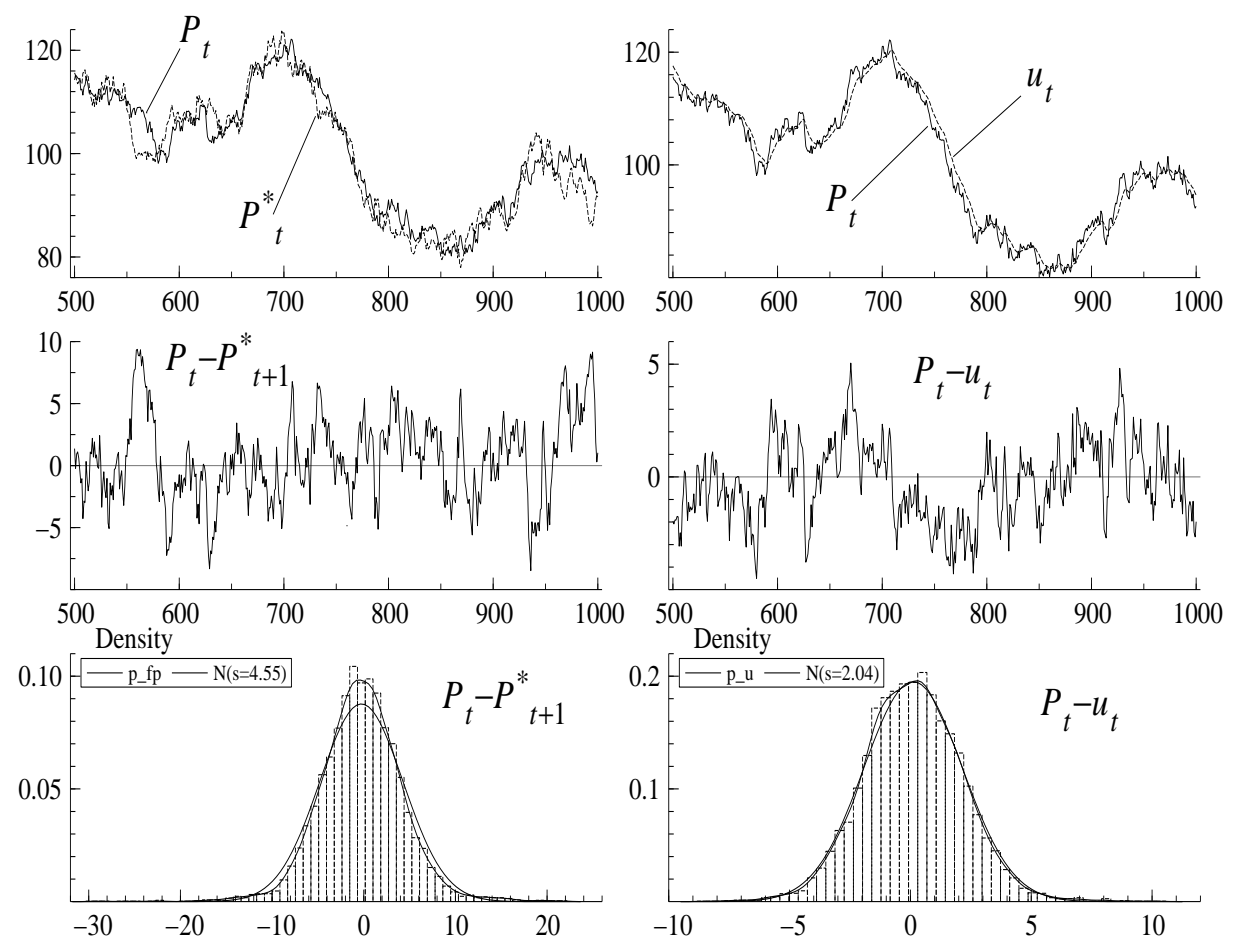

FIgURE 3.4. The price $P_{t}$ and the fundamental price $P_{t}^{*}$ (top left); the geometric moving average $u_{t}$ (top right); the differences $P_{t}-P_{t+1}^{*}$ (middle left) and $P_{t}-u_{t}$ (middle right) and the corresponding distributions (bottom). 
The above result demonstrates that the simple MF model is able to generate realistic price behaviour and appropriate long range dependence for returns when both noise processes are present. Because of the interaction of the nonlinear deterministic dynamics and the noisy processes, an explicit analysis of the generating mechanism for this outcome seems to be difficult in general. In the following, we try to do so from three different aspects ${ }^{15}$.

First, we examine the roles that the two noise processes play on the AC structure of the stochastic system. When there is no noise process, as illustrated in Case-00 in Fig. 3.1, we obtain the underlying deterministic system ${ }^{16}$. The market price is oscillating initially but converging to the fundamental value eventually. When the fundamental price fluctuates stochastically, as in the Case-01, the fundamental values are shifted to different levels over time. In this case, the AC pattern in the top row in Fig. 3.3 shows a significant ACs for returns and highly dependent volatility measured by the significant ACs for the absolute and squared returns. This may be due to the stochastic shift of the fundamental price and the local stability of the underlying deterministic system. When the market price is also perturbed by the noisy excess demand process, the returns display insignificant AC patterns (see the second and third rows in Fig. 3.3). Our simulations show that the two noise processes play different roles. For a given noisy fundamental process with $\sigma_{\epsilon}>0$, there exists a critical value $\sigma_{\delta}^{*}=\sigma_{\delta}^{*}\left(\sigma_{\epsilon}\right)>0$ for the noisy demand process such that the ACs of the returns display a significant pattern for $\sigma_{\delta}<\sigma_{\delta}^{*}$ and an insignificant pattern for $\sigma_{\delta}>\sigma_{\delta}^{*}$. This implies that the noisy demand has a significant impact on the ACs of returns. On the other hand, for a given noisy excess demand with $\sigma_{\delta}>0$, there exists a critical value $\sigma_{\epsilon}^{*}=\sigma_{\epsilon}^{*}\left(\sigma_{\delta}\right)>0$ such that the ACs for the absolute and squared returns display an insignificant pattern for $\sigma_{\epsilon}<\sigma_{\epsilon}^{*}$ and a significant pattern for $\sigma_{\epsilon}>\sigma_{\epsilon}^{*}$. This implies that the noisy fundamental price also has significant impact on the ACs of the absolute and squared returns. Neither one of the two noise processes alone is responsible for the power-law feature.

Second, we examine the impact of the noise processes on the market price and its relation to the fundamental price. The convergence of the market price to the fundamental price for the underlying deterministic system is destroyed after the introduction of the two noise processes, however, the market price moves closely to the fundamental price, as illustrated in the top

\footnotetext{
${ }^{15}$ The authors would like to thank an anonymous referee to bringing some of the following points to our attention.

${ }^{16}$ We refer to He and Li (2005) for the stability and bifurcation analysis in this case.
} 
left panel in Fig. 3.4. This indicates a temporary destabilization of the market price to the fundamental price. From the middle left panel in Fig. 3.4, one can see that such temporary deviation of the market price from the fundamental price can be significant from time to time. However, the density distribution of the differences in the bottom left panel in Fig. 3.4 shows that the market prices are more concentrated near the fundamental prices most of the time. For comparison, we show the relation of the market price and the geometric moving averaged price in the right panels in Fig. 3.4. The moving averaged price is less volatile. Also, its difference from the market price is less concentrated near zero, compared to the difference of the market price from the fundamental price. The reaction of the fundamentalists to the deviation of the market price from the fundamental price and the extrapolation of the trend followers lead to a more realistic price behaviour in this model.

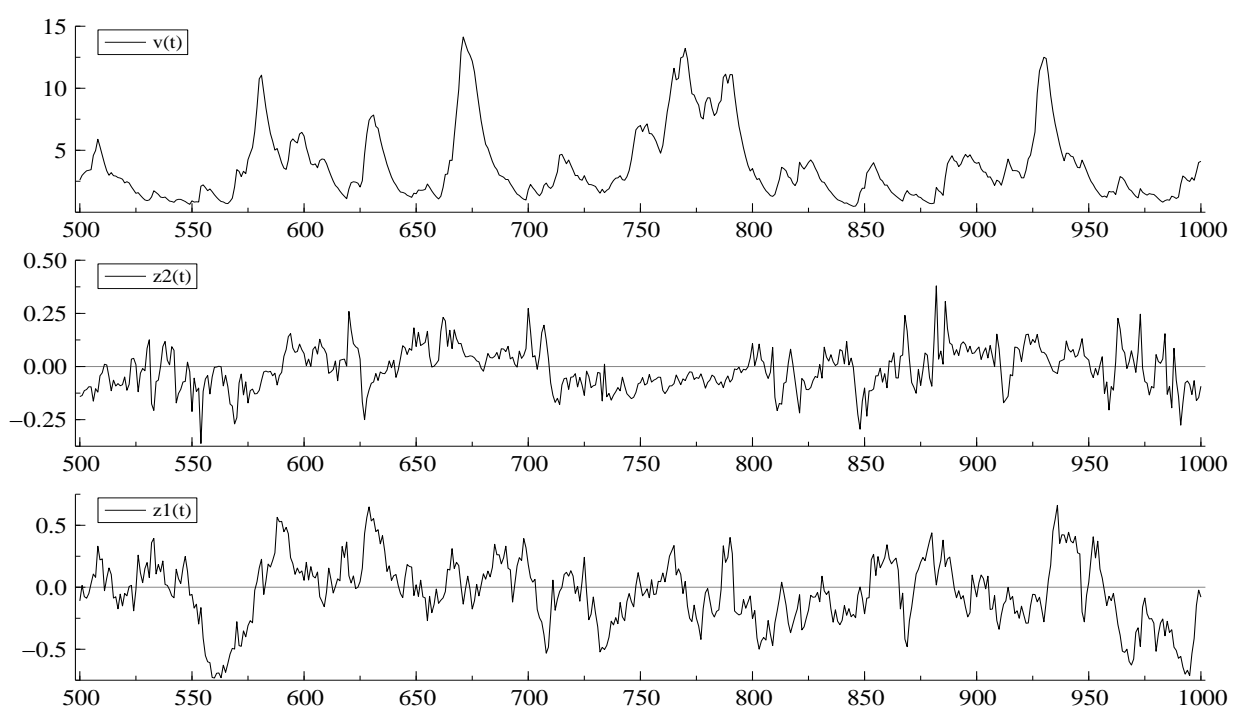

FIGURE 3.5. The geometric volatility ( $v_{t}$, top) and the demands of the trend followers $\left(z_{2 t}\right.$, middle) and the fundamentalists $\left(z_{1 t}\right.$, bottom).

The third important factor possibly affecting the volatility clustering is the endogenous learning process engaged in by the risk averse trend followers. The endogenous development of the expected mean and variance of the trend followers produces a simple feedback effect. The trend followers tend to push the market price away from the fundamental value by extrapolating the trend, leading to high volatility. Because of the perceived increase of risk, their demand/supply is then reduced. The partial withdrawal of the trend followers then leads to less volatile dynamics, which makes the trend followers revise the risk downward so that eventually their demand/supply increases again. This simple feedback mechanism is clearly illustrated in 
Figs. 3.5 and 3.6. In Fig. 3.5, we plot the time series for the geometric moving variance $v_{t}$ (the top panel) and the excess demands of the trend followers $z_{2 t}$ (the middle panel) and of the fundamentalists $z_{1 t}$ (the bottom panel) over a short time period. We have low (high) demand from the trend followers following high (low) perceived volatility. This is further confirmed by the phase plot of $\left(z_{2 t}, v_{t}\right)$ in the left panel in Fig. 3.6 (observe the peaks on either side of zero demand). As expected, the right panel in Fig. 3.6 shows that there is no clear evidence of a relationship between the perceived volatility $\left(v_{t}\right)$ of the trend followers and the excess demand of the fundamentalists.
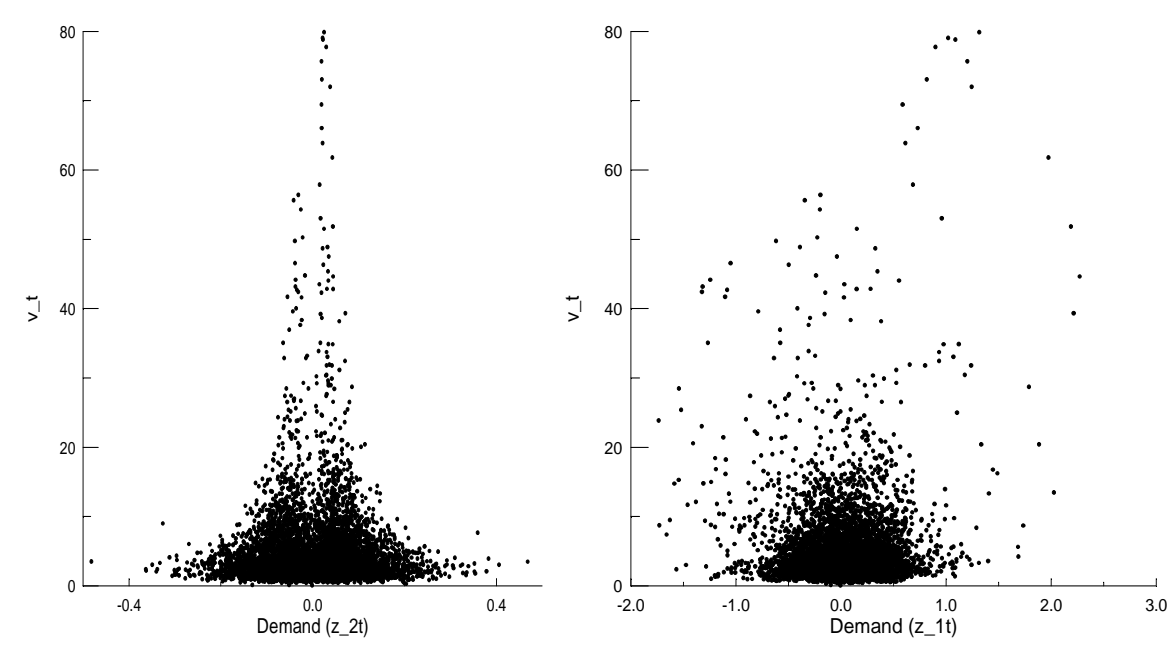

FIGURE 3.6. The phase plots of the geometric moving variance $\left(v_{t}\right)$ and the demands of the trend followers $\left(z_{2 t}\right.$, left $)$ and of the fundamentalists $\left(z_{1 t}\right.$, right $)$.

Overall, we see that the interaction of speculators, the simple feedback of the trend followers, and the interplay of noises and the underlying deterministic dynamics can generate realistic volatility behaviour. We should notice that the size of the noise is a very subtle issue. For the herding mechanism in Lux and Marchesi (2001), a balanced disposition among noise traders is necessary. For the switching mechanism in Gaunersdorfer and Hommes (2006), the noisy component added to the excess demand is responsible for the switching between locally coexisting attractors, and hence the noise level has to be large to obtain realistic results. In our model, the distributed fluctuations are due to the lagged learning and risk adjusted extrapolation from the trend followers need to be balanced by the noise level of the excess demand. At this stage, a theoretical analysis of the interplay of deterministic dynamics and noise seems difficult. Our analysis indicates that the noisy demand plays a more important role in the insignificant 
AC patterns for the returns, while the noisy fundamental process plays a more important role on the significant $\mathrm{AC}$ patterns for the absolute and squared returns.

In the following discussion, we adopt statistical methods based on Monte Carlo simulation to estimate various models related to a power-law characterization. The estimates are obtained for both the MF model and the actual data, including the DAX 30, the FTSE 100, the NIKKEI 225 and the S\&P 500. We also compare the estimates from the actual data with those from the MF model.

\section{Empirical Evidence and Power Law Behaviour of the Actual Data}

This section provides a brief statistical analysis of the DAX 30, the FTSE 100, the NIKKEI 225 , and the S\&P 500 price indices from Datastream, which will be used as empirical evidence and benchmarks for our comparison. There are altogether 5306 daily observations for each index which start from Feb 1st, 1984. Use $p_{t}$ to denote the price index, e.g. the S\&P 500, at time $t(t=0, \ldots, 5305)$ and $\log$ returns $r_{t}$ are defined as $r_{t}=\ln p_{t}-\ln p_{t-1}$.

4.1. Statistics and Autocorrelations of Returns. Table A.1 in Appendix A gives the summary statistics of $r_{t}$ for the DAX 30, the FTSE 100, the NIKKEI 225, and the S\&P 500. For each index, we can see from Table A.1 that the kurtosis for $r_{t}$ is much higher than that of a normal distribution. The kurtosis and studentized range statistics (which is the range divided by the standard deviation) show the characteristic fat-tailed behaviour compared with a normal distribution. The Jarque-Bera normality test statistic suggests that $r_{t}$ is far from a normal distribution.

Ding et al. (1993) investigate autocorrelations of returns (and their transformations) of the daily S\&P 500 index over the period 1928 to 1991 and find that the absolute returns and the squared returns tend to have very slow decaying autocorrelations and the sample autocorrelations for the absolute returns are greater than those for the squared returns at every lag up to at least 100 lags. This kind of AC feature indicates the long-range dependence or the power-law behaviour in volatility. The autocorrelations plotted in Fig. A.1 in Appendix A coincide with the findings in Ding et al. (1993).

4.2. Estimates of Power-Law Decay Index. Besides the visual inspection of autocorrelations of $r_{t}, r_{t}^{2}$ and $\left|r_{t}\right|$, one can also construct models to estimate the decay rate of the autocorrelations 
of $r_{t}, r_{t}^{2}$ and $\left|r_{t}\right|$. For instance, we can semi-parametrically model power-law behaviour in a covariance stationary series $x_{t}, t=0, \pm 1, \ldots$, by

$$
s(\omega) \approx c_{1} \omega^{-2 d}, \omega \rightarrow 0^{+}
$$

where $0<c_{1}<\infty, s(\omega)$ is the spectral density of $x_{t}$, and $\omega$ is the frequency. Under (4.1), $s(\omega)$ has a pole at $\omega=0$ for $0<d<1 / 2$ (when there is a power-law in $x_{t}$ ), while $d \geq 1 / 2$ implies the process is not covariance stationary; $s(\omega)$ is positive and finite for $d=0$; for $-1 / 2<d<0$, we have short and negative dependence, or antipersistence. The ACs can be described by $\rho_{k} \approx c_{2} k^{2 d-1}$, where $c_{2}$ is a constant and $\mu \equiv 2 d-1$ corresponds to the hyperbolic decay index.

Geweke and Poter-Hudak (1983), henceforth GPH, suggest a semiparametric estimator of the fractional differencing parameter, $d$, that is based on a regression of the ordinates of the $\log$ spectral density. Given spectral ordinates $\omega_{j}=2 \pi j / T(j=1,2, \ldots, m)$, GPH suggest estimating $d$ from a regression of the ordinates from the periodogram $I\left(\omega_{j}\right)$. Hence, for $j=$ $1,2, \ldots, m$,

$$
\log I\left(\omega_{j}\right)=c-d \log \left(4 \sin ^{2}\left(\omega_{j} / 2\right)\right)+v_{j}
$$

where $v_{j}$ is assumed to be i.i.d. with zero mean and variance $\pi^{2} / 6$. If the number of ordinates $m$ is chosen such that $m=g(T)$, where $g(T)$ is such that $\lim _{T \rightarrow \infty} g(T)=\infty, \lim _{T \rightarrow \infty} g(T) / T=$ 0 and $\lim _{T \rightarrow \infty}\left(\log (T)^{2}\right) / g(T)=0$, then the OLS estimator of $d$ based on (4.2) has the limiting distribution

$$
\sqrt{m}\left(\hat{d}_{G P H}-d\right) \stackrel{d}{\rightarrow} \mathcal{N}\left(0, \frac{\pi^{2}}{24}\right)
$$

Robinson (1995) provides a formal proof for $-1 / 2<d<1 / 2$, Velasco (1999) proves the consistency of $\hat{d}_{G P H}$ in the case $1 / 2 \leq d<1$ and its asymptotic normality in the case $1 / 2 \leq$ $d<3 / 4$. It is clear from this result that the GPH estimator is not $T^{1 / 2}$ consistent and will converge at a slower rate.

Another most often used estimator of $d$ is developed by Robinson and Henry (1999), henceforth RH. They suggest a semiparametric Gaussian estimate of the memory parameter $d$, by considering

$$
\hat{d}_{R H}=\arg \min _{d} R(d), \quad R(d)=\log \left\{\frac{1}{m} \sum_{j=1}^{m} \omega_{j}^{2 d} I\left(\omega_{j}\right)\right\}-2 \frac{d}{m} \sum_{j=1}^{m} \log \omega_{j},
$$


in which $m \in(0,[T / 2])$. They prove that $\sqrt{m}\left(\hat{d}_{R H}-d\right) \stackrel{d}{\rightarrow} \mathcal{N}\left(0, \frac{1}{4}\right)$ when $m<[T / 2]$ such that $1 / m+m / T \rightarrow 0$ as $T \rightarrow \infty$ and under some further conditions (see Robinson and Henry (1999)).

A major issue in the application of the GPH and the RH estimators is the choice of $m$, due to the fact that some limited knowledge is now available concerning this issue (see, Geweke (1998), for instance), it is a wise precaution to report the estimated results for a range of bandwidths. So in our study, for both the GPH and the RH estimates of $d$, we report the corresponding estimates for $m=50,100,150,200$, and 250, respectively, in Appendix A.

For instance, for the DAX 300, Table A.2 reports the GPH and the RH estimates of $d$ for returns, the squared returns, and the absolute returns, respectively. In the panel of $r_{t}$ in Table A.2, the first row reports the results from the GPH and the RH estimates with $m=50$, the second row reports the results of the GPH and the RH estimates with $m=100$, and so on. This also holds for the panels of $r_{t}^{2}$ and $\left|r_{t}\right|$, and for other tables in this section. The estimates of the parameter $d$ for the FTSE 100, the NIKKEI 225 and the S\&P 500 are summarized in Tables A.3, A.4, and A.5, respectively.

We see that all of the estimated $d$ for the returns are not significant at all conventional significance levels while those for the squared returns, and the absolute returns are significant. Thus, for the DAX 30, the FTSE 100, the NIKKEI 225 and the S\&P 500, there is clear evidence of power-law for the squared and the absolute returns where $d$ is positive, and the persistence in the absolute returns is much stronger than that in the squared returns. These results coincide with the well-established findings in the empirical finance literature.

\subsection{Volatility Clustering, Power-Law and (FI)GARCH Estimates. Another striking fea-} ture of the return series is volatility clustering. A number of econometric models of changing conditional variance have been developed to test and measure volatility clustering. Engle (1982) suggested a test where the null hypothesis is that the residuals of a regression model are i.i.d. and the alternative hypothesis is that the errors are $\operatorname{ARCH}(q)$. Suppose the stock returns follow an $\operatorname{AR}(1)$ process with innovations $\varepsilon_{t}$. If the returns are homoscedastic, then the variance cannot be predicted and the variations in $\varepsilon_{t}^{2}$ will be purely random. However, if ARCH effects are present, large values of $\varepsilon_{t}^{2}$ will be predicted by large values of the past squared residuals. This leads to a $T R^{2}$ test statistic. In order to compute the test statistic, we first fit the returns series 
with an $\operatorname{AR}(1)$ model, and then regress the squared residuals $\varepsilon_{t}^{2}$ on a constant and $\varepsilon_{t-1}^{2}, \ldots, \varepsilon_{t-q}^{2}$. The $R^{2}$ is then computed from this regression. Under the null hypothesis that there is no ARCH, the test statistic is asymptotically distributed as a chi-square distribution with $q$ degrees of freedom. We implement the test for the four indices and the results are reported in Table A.6 in Appendix A. In all the cases, the null hypothesis is strongly rejected and, in terms of Engle's test, the four indices do have clear ARCH effects.

We now consider the family of ARCH models. The most widely used one is that introduced by Engle (1982) and its generalization, the GARCH model, introduced by Bollerslev (1986). Following their specification, for instance, if we model the returns as an AR(1) process, then a $\operatorname{GARCH}(p, q)$ model is defined by:

$$
\left\{\begin{array}{l}
r_{t}=a+b r_{t-1}+\varepsilon_{t}, \quad \varepsilon_{t}=\sigma_{t} z_{t}, \\
\sigma_{t}^{2}=\alpha_{0}+\alpha(L) \varepsilon_{t}^{2}+\beta(L) \sigma_{t}^{2}, \quad z_{t} \sim N(0,1),
\end{array}\right.
$$

where $L$ is the lag operator, $\alpha(L)=\sum_{i=1}^{q} \alpha_{i} L^{i}$ and $\beta(L)=\sum_{j=1}^{p} \beta_{i} L^{j}$. Defining $v_{t}=\varepsilon_{t}^{2}-\sigma_{t}^{2}$, the process can be rewritten as an $\operatorname{ARMA}(m, p)$ process

$$
[1-\alpha(L)-\beta(L)] \varepsilon_{t}^{2}=\alpha_{0}+[1-\beta(L)] v_{t}
$$

with $m=\max \{p, q\}$. Table A.7 in Appendix A reports the estimates of the $\operatorname{GARCH}(1,1)$ model, where the mean process follows an AR(1) structure.

Based on the estimates, one can see that a small influence of the most recent innovation (small $\alpha_{1}$ ) is accompanied by a strong persistence of the variance coefficient (large $\beta_{1}$ ). It is also interesting to observe that the sum of the coefficients $\alpha_{1}+\beta_{1}$ is close to one, which indicates that the process is close to an integrated GARCH (IGARCH) process. Such parameter estimates are rather common when considering returns from high frequency daily financial data of both share and foreign exchange markets (see, Pagan (1996)). The GARCH implies that shocks to the conditional variance decay exponentially. However the IGARCH implies that the shocks to the conditional variance persist indefinitely.

In response to the finding that most financial time series are power-law volatility processes, Baillie et al. (1996) consider the Fractional Integrated GARCH (FIGARCH) process, where a shock to the conditional variance dies out at a slow hyperbolic rate. Later on, Chung (1999) 
suggests a slightly different parameterization of the model:

$$
\phi(L)(1-L)^{d}\left(\varepsilon_{t}^{2}-\sigma^{2}\right)=\alpha_{0}+[1-\beta(L)] v_{t},
$$

where $\phi(L)=1-\sum_{i=1}^{q} \phi_{i} L^{i}, \alpha_{0}=\phi(L)(1-L)^{d} \sigma^{2}$, and $\sigma^{2}$ is the unconditional variance of the corresponding GARCH model. Table A.8 in Appendix A reports the estimates of the FIGARCH $(1, d, 1)$ model, where the mean process follows an $\mathrm{AR}(1)$ model. The estimate for the fractional differencing parameter $\hat{d}$ is statistically very different from both zero and one. This is consistent with the well known findings that the shocks to the conditional variance die out at a slow hyperbolic rate.

\section{Econometric Characterization of The Power-Law Properties of The MF MODEL}

This section is devoted to an econometric analysis on the power-law behaviour and the volatility persistence of the MF model. Various models are estimated using the MF model-generated data outlined in Section 3 and then compared with those of the DAX 30, the FTSE 100, the NIKKEI 225, and the S\&P 500 estimated in the previous section. The analysis and estimates are based on Monte Carlo simulations. For the chosen set of parameters and two noise processes specified in Case-11 in Section 3, we ran 1,000 independent simulations over 6,306 time periods and discarded the first 1,000 time periods to wash out any possible initial noise effect. For each run of the model we have 5,306 observations, which matches the sample size of the actual data that we used in the previous section.

5.1. Autocorrelations of Returns. First, we look at the ACs of returns, the squared returns and the absolute returns. It is interesting to see whether our simulation model can replicate the well known findings as described in Fig. A.1. By running 1,000 independent simulations, we estimate the autocorrelation coefficients and calculate Newey-West corrected standard errors of returns, the squared returns and the absolute returns for each run of the model, and we then take the average. We plot the ACs and their corresponding confidence intervals in Fig. 5.1.

From Fig. 5.1, we see that for the market fraction model, not only are the sample correlations of $r_{t}^{2}$ and $\left|r_{t}\right|$ all outside the $95 \%$ confidence interval of $r_{t}$, but they are also all positive over long lags. Further, the sample autocorrelations for the absolute returns are greater than that for the squared returns at every lag up to at least 100 lags. Comparing with Fig. A.1 for the four 


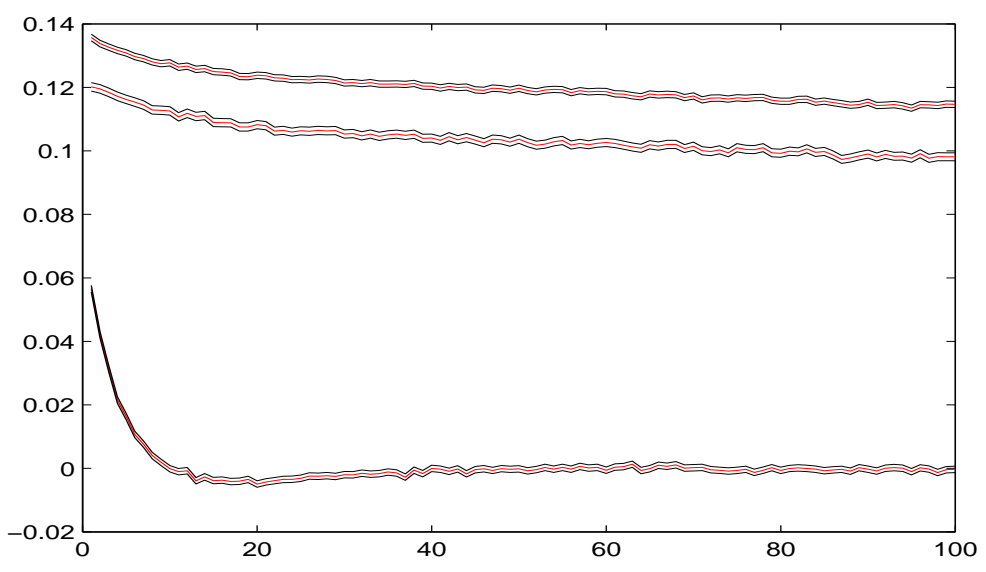

FIGURE 5.1. Autocorrelations of $r_{t}$ (bottom), $r_{t}^{2}$ (middle) and $\left|r_{t}\right|$ (top) for the MF model.

indices, we see that the patterns of decay of the autocorrelation functions of return, the squared return and the absolute return are quite similar.

5.2. Estimates of Power-Law Decay Index. We also look at the decay rate of the autocorrelations of returns, the squared returns, and the absolute returns that are estimated from the GPH the RH method. The resulting estimates are reported in Table A.9 in Appendix A, where the column 'Sig\%' indicates the percentage of simulations for which the corresponding estimates are significant at the 5\% level over 1,000 independent simulations. We find that for $m=50,100$, most of the estimates of $d$ for returns are not significant, but most of them are significant for larger $m$, although the corresponding averaged $p$-values are large. However, all of the estimates of $d$ for the squared returns and the absolute returns are positive. There is a clear evidence of a power-law for the squared returns and the absolute returns, and also the patterns of the estimates of $d$ for the returns, the squared returns, and the absolute returns are comparable to those of the actual data (see Tables A.2, A.3, A.4, and A.5).

\subsection{Volatility Clustering, Power-Law and (FI)GARCH Estimates. We now check for} ARCH/GARCH effects, in order to see whether the MF model is capable of capturing the feature of volatility clustering. We implement the test suggested by Engle (1982). Corresponding to the Table A.6 of such test for the indices, the resulting test statistics for the MF model are $140.8,228.2,372.7,391.8$ and 710.8 with the percentages, $98.7 \%, 99.3 \%, 99.8 \%, 99.9 \%$, and 
$100 \%$, respectively, so that the test statistics are significant at the $5 \%$ level over 1000 independent simulations. In terms of Engle's test, the MF model does have clear ARCH effects. So, we turn to study the GARCH and the FIGARCH estimates that describe volatility persistence.

We report the estimates of the GARCH and the FIGARCH models in Tables A.10 and A.11, respectively. The reported estimates and standard errors are the averages of those across 1000 independent simulations. The specifications of the models are the same as what we estimated for the indices. Again, all these estimates are obtained from the estimates for each run of the simulation model and then averaged over independent simulations. The results from the GARCH model are astonishingly similar to what one usually extracts from real life data: a small influence of the most recent innovation $\left(\alpha_{1}<0.1\right)$ is accompanied by strong persistence of the variance coefficient $\left(\beta_{1}>0.9\right)$ and the sum of the coefficients $\alpha_{1}+\beta_{1}=0.9928$ is close to one. For the estimates of the $\operatorname{FIGARCH}(1, d, 1)$, we see that the estimate of $d$ is significantly different from zero and one.

Overall, we find that the MF model does provide a mechanism that can generate the longrange dependence in volatility observed in actual market data. Now we turn to assess the differences between the MF model and the real world quantitatively.

\subsection{Comparing with the Actual Data in Terms of the Power-Law Characteristics. Here} we compare the MF model with the four indices in terms of the ACs of returns, the squared returns and the absolute returns, the power-law decay index $d$, and the parameter $d$ in the FI$\operatorname{GARCH}(1,1)$ specification, respectively.

In Figures 5.2, we plot the autocorrelation coefficients of returns, the squared returns and the absolute returns for the MF model together with the DAX 30, the FTSE 100, the NIKKEI 225 and the S\&P 500 respectively. For the purpose of comparison, we plot the corresponding confidence intervals for corresponding quantities coming from the actual data.

For the returns, we see from the first column of Fig. 5.2 that all of the autocorrelations of the MF model lies inside the confidence intervals of the actual data. However, for the squared returns, we see from the second column of Fig. 5.2 that while the line of the MF model looks reasonable compared to the DAX 30, it is different from what we see from the S\&P 500. The last column of Fig. 5.2 also provides a mixed picture for the absolute returns, the MF model seems to fit the DAX 30 better than the other indices, especially for large lags. 

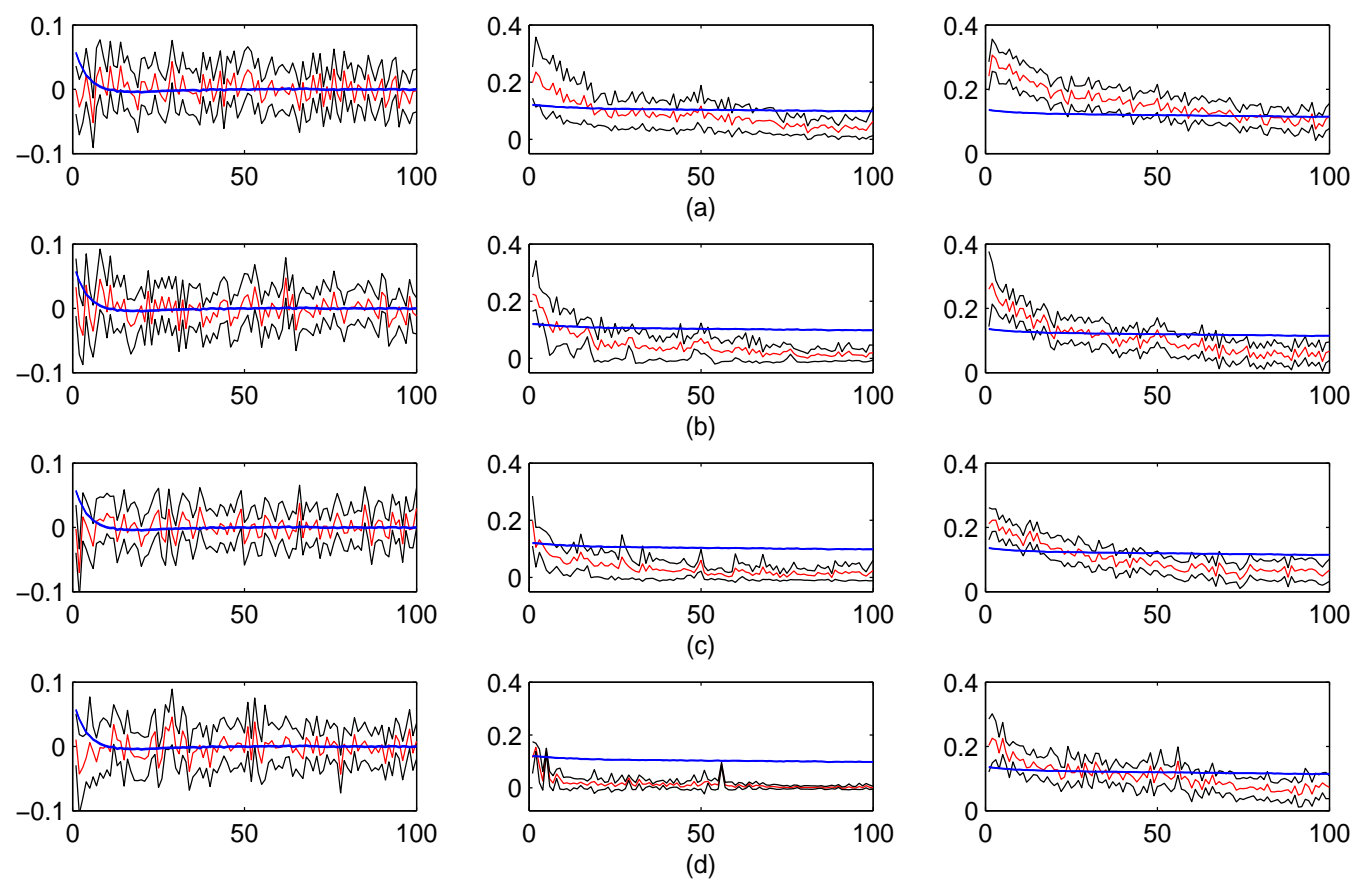

FIGURE 5.2. The ACs of the returns, the squared returns and the absolute returns for the MF model and the DAX 30 (a), the FTSE 100 (b), the NIKKEI 225 (c), and the S\&P 500 (d). The smooth lines refer to the MF model while the confidence intervals are those for the actual data.

For the decay index $d$ of the returns, the squared returns or the absolute returns, we want to test whether the parameters $d$ estimated from both the actual data (for instance, the DAX 30) and the MF model are the same. In other words, we want to test $H_{0}: d_{D A X}=d_{M F}$, apart from checking whether $\hat{d}_{M F}$ lies in the confidence interval of $\hat{d}_{D A X}$ or not. The null hypothesis can also be tested by the Wald test by assuming that both the number of simulations and the number of time periods for each simulation go to infinity. In the construction of the Wald test, $W=\left(\hat{d}_{D A X}-\hat{d}_{M F}\right) \hat{\Sigma}^{-1}\left(\hat{d}_{D A X}-\hat{d}_{M F}\right)$, where $\hat{\Sigma}$ is simply the variance of $\hat{d}_{D A X}$. We notice that $d_{M F}$ is estimated from the simulated data by running the MF model independently many times, so $\hat{d}_{M F}$ converges much faster than that of $\hat{d}_{D A X}$ and we can ignore the estimation inaccuracy in $\hat{d}_{M F}$. For a more general discussion on a comparison of simulation models with the real world data, see Li et al. (2006a, 2006b). The resulting test statistics are summarized in Table A.12 in Appendix A, in the column 'DAX 30', the first sub-column reports the test statistics corresponding to $\hat{d}_{G P H}$, and the second sub-column corresponding to $\hat{d}_{R H}$, and so on. Notice that the critical values of the Wald test at $5 \%$ and $1 \%$ significant levels are 3.842 and 6.635 , respectively. For the returns, we see that the estimated $d$ of the FTSE 100 and the MF model are 
not significantly different, except for the RH estimate with $m=250$; in addition, most of the test statistics are not significant for $m=50,100$, but they are significant for larger $m$. For the squared returns, except for the GPH estimates of the DAX 30, the estimated $d$ of the MF model is significantly different from almost all of those of the actual data. For the absolute returns, the differences between the estimated $d$ of the actual data and the MF model are not statistically significant, except for the $m=50$.

We can also test the equality of $d$ in the FIGARCH specification between the actual data and the MF model by the Wald test. The resulting Wald statistics for the DAX 30, FTSE 100, the NIKKEI 225 and the S\&P 500 are 586, 4.140, 29.5 and 4.853, respectively. So the estimated $d$ in the FIGARCH $(1, d, 1)$ model of the MF model is not significantly different from those of the FTSE 100 and the S\&P 500, but it is significantly different from those of the DAX 30 and the NIKKEI 225.

The above analysis indicates that the simple market fraction model is able to replicate the power-law properties of the actual stock market qualitatively. However, the formal statistical tests find that the decay rate and the (FI)GARCH estimates from the MF model do not easily completely match those of any particular single index ${ }^{17}$. This is probably due to the simplicity of the MF model and different features across different financial markets. The power-law mechanism of the MF model is different from either herding (for instance, the mechanism developed in Lux and Marchesi (1999)) or switching mechanisms (such as the adaptive switching mechanism in Brock and Hommes $(1997,1998))$ in terms of modeling, but it shares the same spirit in a much simpler way. This is one of the main contributions of this paper. It is this simplicity that makes it possible to identify potential sources and mechanisms that can generate certain characteristics.

\section{Conclusion}

Motivated by the recent interest in the power-law behaviour of high frequency financial market time series and the explanatory power of heterogeneous-agent asset-pricing models, this paper investigates the power-law properties of a simple market fraction model involving two types of traders (fundamentalists and trend followers). Extending earlier work on long-run asset price behaviour, profitability, survivability, various under- and over-reaction AC patterns,

\footnotetext{
${ }^{17}$ This is not too surprising, we might note that these parameters also differ across stock indices.
} 
and their connections to the underlying deterministic dynamics, we studied in the characterization of the power-law volatility behaviour of the MF model and its comparison with the real world. We found that the agent heterogeneity, risk-adjusted trend chasing through the geometric learning process, and the interplay of noise and the underlying deterministic dynamics can explain the power-law distributed fluctuations.

It is interesting and important to see how the deterministic dynamics and noise interact with each other, and further, to understand the connections between the nonlinear dynamics of the underlying deterministic system and certain time series properties of the corresponding stochastic system. The theoretical analysis is important but difficult given the current state of knowledge on nonlinear random dynamic system. Therefore statistical analysis with powerful econometric tools seems necessary. Based upon Monte Carlo simulations, statistical analysis, including estimates of the (FI)GARCH parameters and related tests, we show that the MF model is able to explain some of the characteristics that are well established in the empirical finance literature. There is a clear evidence of the power-law and GARCH effects. It is worth emphasizing that all these interesting qualitative and quantitative features arise from the simple model with fixed market fractions.

Further investigation and extension of the simple model seems necessary. It may be interesting to extend our analysis to the model established recently by Dieci et al. (2006), in which part of the market fractions are governed by market mood and the rest follows some adaptive switching process. One way to start might be to estimate the model first, and then implement misspecification tests. Econometric methods, such as efficient methods of moments could be used. Allowing for market mood and switching mechanisms and using these econometric estimation approaches, we may gain a better characterization and understanding of the mechanisms deriving financial markets.

Appendix A. Statistical Results

TABLE A.1. Summary statistics of $r_{t}$.

\begin{tabular}{lcccccccc}
\hline \multicolumn{1}{c}{ data } & mean & std. & skewness & kurtosis & min & max & stud. range & Jarque-Bera \\
\hline DAX 30 & 0.0003 & 0.0143 & -0.467 & 8.940 & -0.137 & 0.076 & 14.91 & 7991 \\
FTSE 100 & 0.0003 & 0.0105 & -0.735 & 13.07 & -0.130 & 0.076 & 19.60 & 22879 \\
NIKKEI 225 & 0.0000 & 0.0137 & -0.142 & 10.47 & -0.161 & 0.124 & 20.78 & 12365 \\
S\&P 500 & 0.0004 & 0.0107 & -1.997 & 45.96 & -0.228 & 0.087 & 29.35 & 411423 \\
\hline
\end{tabular}




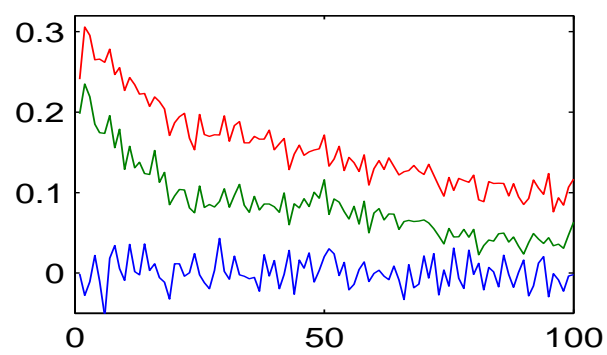

(a)

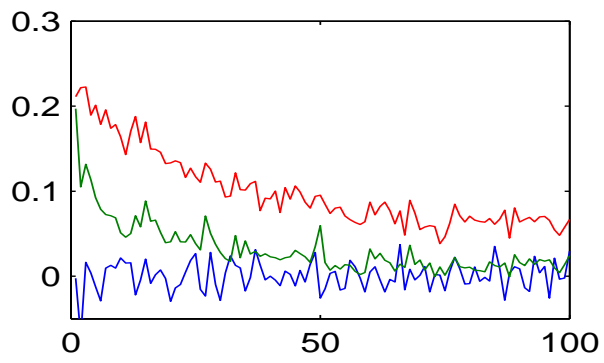

(c)

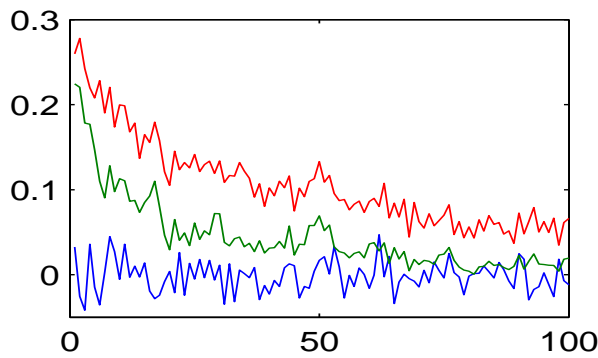

(b)

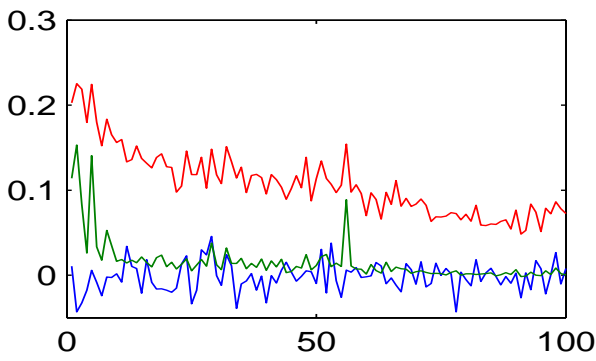

(d)

FIGURE A.1. Autocorrelations of returns, the squared returns and the absolute returns for the DAX 30 (a), the FTSE 100 (b), the NIKKEI 225 (c), and the S\&P 500 (d). The lines from the bottom to the top are the autocorrelations for returns, the squared returns, and the absolute returns, respectively.

TABle A.2. The estimates of $d$ for the DAX 30 with $m=50,100,150,200,250$

\begin{tabular}{ccccc||cccc}
\hline & $\hat{d}_{G P H}$ & $t$ & $p$-value & $95 \%$ CI & $\hat{d}_{R H}$ & $t$ & $p$-value & $95 \%$ CI \\
\hline$r_{t}$ & 0.0884 & 0.858 & 0.391 & {$[-0.1136,0.2903]$} & -0.0034 & -0.048 & 0.962 & {$[-0.1420,0.1352]$} \\
& 0.0491 & 0.707 & 0.480 & {$[-0.0870,0.1852]$} & 0.0634 & 1.267 & 0.205 & {$[-0.0346,0.1614]$} \\
& 0.0527 & 0.948 & 0.343 & {$[-0.0563,0.1617]$} & 0.0901 & 2.208 & 0.027 & {$[0.0101,0.1702]$} \\
& 0.0348 & 0.730 & 0.465 & {$[-0.0586,0.1281]$} & 0.0528 & 1.493 & 0.135 & {$[-0.0165,0.1221]$} \\
& 0.0434 & 1.027 & 0.305 & {$[-0.0395,0.1264]$} & 0.0609 & 1.926 & 0.054 & {$[-0.0011,0.1229]$} \\
\hline$r_{t}^{2}$ & 0.4380 & 4.252 & 0.000 & {$[0.2361,0.6400]$} & 0.4156 & 5.878 & 0.000 & {$[0.2770,0.5542]$} \\
& 0.4727 & 6.807 & 0.000 & {$[0.3366,0.6089]$} & 0.4570 & 9.139 & 0.000 & {$[0.3590,0.5550]$} \\
& 0.4111 & 7.391 & 0.000 & {$[0.3021,0.5201]$} & 0.3887 & 9.521 & 0.000 & {$[0.3087,0.4687]$} \\
& 0.3710 & 7.787 & 0.000 & {$[0.2776,0.4643]$} & 0.3649 & 10.32 & 0.000 & {$[0.2956,0.4342]$} \\
& 0.3830 & 9.054 & 0.000 & {$[0.3001,0.4660]$} & 0.3714 & 11.74 & 0.000 & {$[0.3094,0.4334]$} \\
\hline$\left|r_{t}\right|$ & 0.6478 & 6.287 & 0.000 & {$[0.4458,0.8497]$} & 0.6137 & 8.678 & 0.000 & {$[0.4751,0.7522]$} \\
& 0.6013 & 8.658 & 0.000 & {$[0.4652,0.7374]$} & 0.5986 & 11.97 & 0.000 & {$[0.5006,0.6966]$} \\
& 0.5846 & 10.51 & 0.000 & {$[0.4756,0.6936]$} & 0.5565 & 13.63 & 0.000 & {$[0.4765,0.6366]$} \\
& 0.5404 & 11.34 & 0.000 & {$[0.4471,0.6338]$} & 0.5366 & 15.18 & 0.000 & {$[0.4673,0.6059]$} \\
& 0.5215 & 12.33 & 0.000 & {$[0.4386,0.6044]$} & 0.5166 & 16.34 & 0.000 & {$[0.4546,0.5785]$} \\
\hline
\end{tabular}


TABLE A.3. The estimates of $d$ for the FTSE 100 with $m=50,100,150,200,250$

\begin{tabular}{ccccc||cccc}
\hline & $\hat{d}_{G P H}$ & $t$ & $p$-value & $95 \%$ CI & $\hat{d}_{R H}$ & $t$ & $p$-value & 95\% CI \\
\hline$r_{t}$ & -0.0641 & -0.623 & 0.534 & {$[-0.2661,0.1378]$} & 0.0109 & 0.155 & 0.877 & {$[-0.1277,0.1495]$} \\
& -0.0560 & -0.806 & 0.420 & {$[-0.1921,0.0801]$} & -0.0174 & -0.349 & 0.727 & {$[-0.1154,0.0806]$} \\
& -0.0881 & -1.582 & 0.114 & {$[-0.1972,0.0211]$} & -0.0410 & -1.004 & 0.315 & {$[-0.1210,0.0390]$} \\
& -0.0695 & -1.458 & 0.145 & {$[-0.1628,0.0239]$} & -0.0289 & -0.817 & 0.414 & {$[-0.0982,0.0404]$} \\
& -0.0178 & -0.421 & 0.673 & {$[-0.1008,0.0651]$} & -0.0051 & -0.162 & 0.871 & {$[-0.0671,0.0569]$} \\
\hline$r_{t}^{2}$ & 0.2739 & 2.658 & 0.008 & {$[0.0719,0.4758]$} & 0.2958 & 4.184 & 0.000 & {$[0.1572,0.4344]$} \\
& 0.2802 & 4.035 & 0.000 & {$[0.1441,0.4164]$} & 0.2923 & 5.845 & 0.000 & {$[0.1943,0.3903]$} \\
& 0.2469 & 4.439 & 0.000 & {$[0.1379,0.3559]$} & 0.2684 & 6.575 & 0.000 & {$[0.1884,0.3485]$} \\
& 0.2201 & 4.621 & 0.000 & {$[0.1268,0.3135]$} & 0.2560 & 7.239 & 0.000 & {$[0.1867,0.3253]$} \\
& 0.2297 & 5.430 & 0.000 & {$[0.1468,0.3126]$} & 0.2630 & 8.318 & 0.000 & {$[0.2011,0.3250]$} \\
\hline$\left|r_{t}\right|$ & 0.5609 & 5.444 & 0.000 & {$[0.3589,0.7628]$} & 0.5797 & 8.197 & 0.000 & {$[0.4411,0.7182]$} \\
& 0.6044 & 8.702 & 0.000 & {$[0.4682,0.7405]$} & 0.5666 & 11.33 & 0.000 & {$[0.4686,0.6646]$} \\
& 0.5656 & 10.17 & 0.000 & {$[0.4566,0.6746]$} & 0.5242 & 12.84 & 0.000 & {$[0.4441,0.6042]$} \\
& 0.5156 & 10.82 & 0.000 & {$[0.4222,0.6089]$} & 0.5023 & 14.21 & 0.000 & {$[0.4330,0.5716]$} \\
& 0.5134 & 12.13 & 0.000 & {$[0.4305,0.5963]$} & 0.5003 & 15.82 & 0.000 & {$[0.4384,0.5623]$} \\
\hline
\end{tabular}

TABLE A.4. The estimates of $d$ for the NIKKEI 225 with $m=50,100,150,200,250$

\begin{tabular}{ccccc||cccc}
\hline & $\hat{d}_{G P H}$ & $t$ & $p$-value & $95 \%$ CI & $\hat{d}_{R H}$ & $t$ & $p$-value & $95 \%$ CI \\
\hline$r_{t}$ & 0.1560 & 1.514 & 0.130 & {$[-0.0460,0.3579]$} & 0.0476 & 0.673 & 0.501 & {$[-0.0910,0.1862]$} \\
& 0.1107 & 1.594 & 0.111 & {$[-0.0254,0.2469]$} & 0.0411 & 0.821 & 0.411 & {$[-0.0569,0.1391]$} \\
& 0.0796 & 1.431 & 0.152 & {$[-0.0294,0.1886]$} & 0.0483 & 1.183 & 0.237 & {$[-0.0317,0.1283]$} \\
& 0.0518 & 1.088 & 0.277 & {$[-0.0415,0.1452]$} & 0.0347 & 0.981 & 0.326 & {$[-0.0346,0.1040]$} \\
& 0.0283 & 0.668 & 0.504 & {$[-0.0547,0.1112]$} & 0.0127 & 0.403 & 0.687 & {$[-0.0493,0.0747]$} \\
\hline$r_{t}^{2}$ & 0.3277 & 3.180 & 0.001 & {$[0.1257,0.5296]$} & 0.3746 & 5.298 & 0.000 & {$[0.2361,0.5132]$} \\
& 0.3251 & 4.681 & 0.000 & {$[0.1890,0.4612]$} & 0.3250 & 6.500 & 0.000 & {$[0.2270,0.4230]$} \\
& 0.3231 & 5.810 & 0.000 & {$[0.2141,0.4321]$} & 0.3145 & 7.704 & 0.000 & {$[0.2345,0.3945]$} \\
& 0.3211 & 6.740 & 0.000 & {$[0.2277,0.4145]$} & 0.3164 & 8.948 & 0.000 & {$[0.2471,0.3857]$} \\
& 0.3147 & 7.437 & 0.000 & {$[0.2317,0.3976]$} & 0.3059 & 9.673 & 0.000 & {$[0.2439,0.3679]$} \\
\hline$\left|r_{t}\right|$ & 0.6019 & 5.841 & 0.000 & {$[0.3999,0.8038]$} & 0.6060 & 8.570 & 0.000 & {$[0.4674,0.7446]$} \\
& 0.5174 & 7.449 & 0.000 & {$[0.3812,0.6535]$} & 0.5270 & 10.54 & 0.000 & {$[0.4290,0.6250]$} \\
& 0.5356 & 9.631 & 0.000 & {$[0.4266,0.6446]$} & 0.5193 & 12.72 & 0.000 & {$[0.4393,0.5993]$} \\
& 0.5103 & 10.71 & 0.000 & {$[0.4169,0.6037]$} & 0.5112 & 14.46 & 0.000 & {$[0.4419,0.5805]$} \\
& 0.5121 & 12.11 & 0.000 & {$[0.4292,0.5951]$} & 0.5090 & 16.09 & 0.000 & {$[0.4470,0.5709]$} \\
\hline
\end{tabular}

TABLE A.5. The estimates of $d$ for the S\&P 500 with $m=50,100,150,200,250$

\begin{tabular}{ccccc||cccc}
\hline & $\hat{d}_{G P H}$ & $t$ & $p$-value & $95 \%$ CI & $\hat{d}_{R H}$ & $t$ & $p$-value & $95 \%$ CI \\
\hline$r_{t}$ & 0.0606 & 0.588 & 0.557 & {$[-0.1414,0.2625]$} & 0.0575 & 0.814 & 0.416 & {$[-0.0811,0.1961]$} \\
& 0.0375 & 0.537 & 0.591 & {$[-0.0994,0.1744]$} & 0.0306 & 0.612 & 0.541 & {$[-0.0674,0.1286]$} \\
& 0.0287 & 0.515 & 0.606 & {$[-0.0804,0.1378]$} & 0.0137 & 0.335 & 0.737 & {$[-0.0663,0.0937]$} \\
& 0.0232 & 0.488 & 0.626 & {$[-0.0701,0.1166]$} & 0.0028 & 0.078 & 0.938 & {$[-0.0665,0.0721]$} \\
& 0.0155 & 0.367 & 0.714 & {$[-0.0674,0.0985]$} & -0.0023 & -0.072 & 0.943 & {$[-0.0643,0.0597]$} \\
\hline$r_{t}^{2}$ & 0.2425 & 2.354 & 0.018 & {$[0.0406,0.4445]$} & 0.2558 & 3.618 & 0.000 & {$[0.1172,0.3944]$} \\
& 0.2249 & 3.239 & 0.001 & {$[0.0888,0.3611]$} & 0.2455 & 4.909 & 0.000 & {$[0.1475,0.3435]$} \\
& 0.1707 & 3.070 & 0.002 & {$[0.0617,0.2797]$} & 0.1905 & 4.667 & 0.000 & {$[0.1105,0.2706]$} \\
& 0.1493 & 3.133 & 0.002 & {$[0.0559,0.2426]$} & 0.1732 & 4.899 & 0.000 & {$[0.1039,0.2425]$} \\
& 0.1418 & 3.351 & 0.001 & {$[0.0589,0.2247]$} & 0.1700 & 5.374 & 0.000 & {$[0.1080,0.2319]$} \\
\hline$\left|r_{t}\right|$ & 0.6241 & 6.057 & 0.000 & {$[0.4221,0.8260]$} & 0.6139 & 8.682 & 0.000 & {$[0.4753,0.7525]$} \\
& 0.6096 & 8.778 & 0.000 & {$[0.4735,0.7458]$} & 0.6084 & 12.17 & 0.000 & {$[0.5104,0.7064]$} \\
& 0.5530 & 9.943 & 0.000 & {$[0.4440,0.6620]$} & 0.5152 & 12.62 & 0.000 & {$[0.4352,0.5952]$} \\
& 0.4888 & 10.26 & 0.000 & {$[0.3954,0.5822]$} & 0.4856 & 13.74 & 0.000 & {$[0.4163,0.5549]$} \\
& 0.4515 & 10.67 & 0.000 & {$[0.3686,0.5344]$} & 0.4659 & 14.73 & 0.000 & {$[0.4039,0.5279]$} \\
\hline
\end{tabular}


TABLE A.6. Engle's test statistics for the presence of ARCH/GARCH effects

\begin{tabular}{lccccc}
\hline \multicolumn{1}{c}{ data } & lag 1 & lag 2 & lag 5 & lag 10 & lag 50 \\
\hline DAX 30 & 234.5 & 440.9 & 620.5 & 713.4 & 809.1 \\
FTSE 100 & 1415 & 1433 & 1462 & 1497 & 1596 \\
NIKKEI 225 & 240.1 & 261.8 & 347.4 & 364.3 & 438.4 \\
S\&P 500 & 85.65 & 182.8 & 280.5 & 293.6 & 351.7 \\
\hline
\end{tabular}

TABle A.7. GARCH $(1,1)$ Parameter Estimates

\begin{tabular}{lccccc}
\hline \multicolumn{1}{c}{ data } & $a \times 10^{3}$ & $b$ & $\alpha_{0} \times 10^{4}$ & $\alpha_{1}$ & $\beta_{1}$ \\
\hline DAX 30 & $0.655(0.161)$ & $0.0335(0.0162)$ & $0.048(0.004)$ & $0.1185(0.0049)$ & $0.8604(0.0071)$ \\
FTSE 100 & $0.514(0.120)$ & $0.0404(0.0149)$ & $0.023(0.003)$ & $0.0966(0.0066)$ & $0.8824(0.0085)$ \\
NIKKEI 225 & $0.751(0.138)$ & $0.0415(0.0150)$ & $0.023(0.003)$ & $0.1392(0.0036)$ & $0.8608(0.0046)$ \\
S\&P 500 & $0.600(0.119)$ & $0.0267(0.0154)$ & $0.013(0.002)$ & $0.0797(0.0020)$ & $0.9114(0.0036)$ \\
\hline
\end{tabular}

Note: The numbers in parentheses are standard errors. This also holds for Table A.8.

TAble A.8. FigARCH $(1, d, 1)$ Parameter Estimates

\begin{tabular}{lcccccc}
\hline \multicolumn{1}{c}{ data } & $a \times 10^{3}$ & $b$ & $\alpha_{0} \times 10^{4}$ & $\mathrm{~d}$ & $\phi_{1}$ & $\beta$ \\
\hline DAX 30 & $0.694(0.142)$ & $0.0358(0.0144)$ & $0.933(0.057)$ & $0.0675(0.0129)$ & $0.9608(0.0044)$ & $0.9059(0.0088)$ \\
FTSE 100 & $0.528(0.118)$ & $0.0459(0.0143)$ & $0.673(0.093)$ & $0.3270(0.0259)$ & $0.0150(0.0556)$ & $0.2559(0.0739)$ \\
NIKKEI 225 & $20.75(0.070)$ & $-0.0460(0.0010)$ & $0.056(0.024)$ & $0.4047(0.0046)$ & $0.1454(0.0029)$ & $0.7542(0.0027)$ \\
S\&P 500 & $0.629(0.116)$ & $0.0290(0.0158)$ & $0.665(0.094)$ & $0.3353(0.0202)$ & $0.2765(0.0367)$ & $0.5032(0.0447)$
\end{tabular}

TABLE A.9. The estimates of $d$ for the MF model with $m=50,100,150,200,250$

\begin{tabular}{cccccc||ccccc}
\hline & $\hat{d}_{G P H}$ & $t$ & $p$-value & $95 \%$ CI & Sig\% & $\hat{d}_{R H}$ & $t$ & $p$-value & $95 \%$ CI & Sig\% \\
\hline$r_{t}$ & -0.0500 & -0.4856 & 0.4123 & {$[-0.0564,-0.0436]$} & 13.2 & -0.0581 & -0.8215 & 0.3318 & {$[-0.0625,-0.0537]$} & 26.3 \\
& -0.0892 & -1.2842 & 0.2890 & {$[-0.0935,-0.0849]$} & 37.4 & -0.0916 & -1.8311 & 0.2251 & {$[-0.0947,-0.0885]$} & 47.6 \\
& -0.1111 & -1.9976 & 0.2057 & {$[-0.1146,-0.1077]$} & 52.3 & -0.1091 & -2.6722 & 0.1513 & {$[-0.1116,-0.1066]$} & 63.6 \\
& -0.1133 & -2.3783 & 0.1670 & {$[-0.1163,-0.1104]$} & 60.8 & -0.1091 & -3.0862 & 0.1161 & {$[-0.1113,-0.1069]$} & 71.0 \\
& -0.1065 & -2.5173 & 0.1340 & {$[-0.1091,-0.1039]$} & 67.1 & -0.1021 & -3.2294 & 0.1027 & {$[-0.1041,-0.1002]$} & 76.3 \\
\hline$r_{t}^{2}$ & 0.7529 & 7.3072 & 0.0000 & {$[0.7465,0.7593]$} & 100 & 0.7380 & 10.436 & 0.0000 & {$[0.7336,0.7423]$} & 100 \\
& 0.5876 & 8.4603 & 0.0000 & {$[0.5833,0.5919]$} & 100 & 0.5964 & 11.929 & 0.0000 & {$[0.5933,0.5995]$} & 100 \\
& 0.4884 & 8.7822 & 0.0000 & {$[0.4850,0.4919]$} & 100 & 0.5175 & 12.676 & 0.0000 & {$[0.5150,0.5200]$} & 100 \\
& 0.4258 & 8.9378 & 0.0000 & {$[0.4228,0.4288]$} & 100 & 0.4698 & 13.288 & 0.0000 & {$[0.4676,0.4720]$} & 100 \\
& 0.3791 & 8.9610 & 0.0000 & {$[0.3765,0.3818]$} & 100 & 0.4341 & 13.727 & 0.0000 & {$[0.4321,0.4360]$} & 100 \\
\hline$\left|r_{t}\right|$ & 0.8696 & 8.4395 & 0.0000 & {$[0.8632,0.8760]$} & 100 & 0.8519 & 12.048 & 0.0000 & {$[0.8475,0.8563]$} & 100 \\
& 0.7068 & 10.176 & 0.0000 & {$[0.7025,0.7111]$} & 100 & 0.7125 & 14.250 & 0.0000 & {$[0.7094,0.7156]$} & 100 \\
& 0.5975 & 10.741 & 0.0000 & {$[0.5940,0.6009]$} & 100 & 0.6279 & 15.379 & 0.0000 & {$[0.6253,0.6304]$} & 100 \\
& 0.5235 & 10.987 & 0.0000 & {$[0.5205,0.5265]$} & 100 & 0.5731 & 16.208 & 0.0000 & {$[0.5709,0.5753]$} & 100 \\
& 0.4683 & 11.066 & 0.0000 & {$[0.4657,0.4709]$} & 100 & 0.5327 & 16.845 & 0.0000 & {$[0.5307,0.5347]$} & 100 \\
\hline
\end{tabular}


TABle A.10. The GARCH $(1,1)$ Parameter Estimates for the MF Model

\begin{tabular}{ccccc}
\hline$a \times 10^{3}$ & $b$ & $\alpha_{0} \times 10^{4}$ & $\alpha_{1}$ & $\beta$ \\
\hline 0.0740 & 0.0725 & 0.0078 & 0.0260 & 0.9738 \\
$(0.2300)$ & $(0.0139)$ & $(0.0035)$ & $(0.0032)$ & $(0.0033)$ \\
\hline 47 & 77.1 & 17.7 & 100 & 100
\end{tabular}

Note: The numbers in parentheses are the standard errors, and the numbers in the last row are the percentages that the test statistics are significant at $5 \%$ level over 1000 independent simulations. This also holds for Table A.11.

TABle A.11. The FIGARCH $(1, d, 1)$ Parameter Estimates for the MF Model

\begin{tabular}{cccccc}
\hline$a$ & $b$ & $\alpha_{0} \times 10^{4}$ & $\mathrm{~d}$ & $\phi_{1}$ & $\beta$ \\
\hline 0.0137 & 0.0769 & 0.3620 & 0.3797 & 0.3439 & 0.7933 \\
$(0.0010)$ & $(0.0195)$ & $(0.6112)$ & $(0.0386)$ & $(0.0281)$ & $(0.0295)$ \\
\hline 41.2 & 72.6 & 35.6 & 87.6 & 83.1 & 98.5
\end{tabular}

TABLE A.12. The Wald test of $d$ with $m=50,100,150,200,250$

\begin{tabular}{ccc|cc|ccccc}
\hline \multicolumn{4}{c}{ DAX 30 } & \multicolumn{2}{c}{ FTSE 100 } & \multicolumn{2}{c}{ NIKKEI 225 } & \multicolumn{2}{c}{ S\&P 500 } \\
\hline$r_{t}$ & 1.806 & 0.599 & 0.019 & 0.953 & 4.000 & 2.235 & 1.153 & 2.674 \\
& 3.960 & 9.610 & 0.228 & 2.202 & 8.273 & 7.044 & 3.323 & 5.973 \\
& 8.679 & 23.84 & 0.171 & 2.786 & 11.76 & 14.88 & 6.322 & 9.059 \\
& 9.681 & 20.92 & 0.847 & 5.133 & 12.03 & 16.50 & 8.223 & 9.992 \\
& 12.56 & 26.61 & 4.397 & 9.423 & 10.16 & 13.20 & 8.318 & 9.974 \\
\hline$r_{t}^{2}$ & 9.347 & 20.79 & 21.63 & 39.12 & 17.04 & 26.42 & 24.56 & 46.52 \\
& 2.733 & 7.773 & 19.56 & 36.99 & 14.27 & 29.46 & 27.23 & 49.25 \\
& 1.933 & 9.966 & 18.87 & 37.28 & 8.839 & 24.76 & 32.65 & 64.24 \\
& 1.325 & 8.781 & 18.67 & 36.48 & 4.838 & 18.78 & 33.74 & 70.20 \\
& 0.009 & 3.937 & 12.47 & 29.32 & 2.318 & 16.46 & 31.47 & 69.85 \\
\hline$\left|r_{t}\right|$ & 4.637 & 11.35 & 8.983 & 14.82 & 6.755 & 12.10 & 5.681 & 11.33 \\
& 2.304 & 2.595 & 2.171 & 4.259 & 7.427 & 6.884 & 1.956 & 2.168 \\
& 0.054 & 1.020 & 0.329 & 2.151 & 1.240 & 2.360 & 0.641 & 2.541 \\
& 0.126 & 0.267 & 0.028 & 1.003 & 0.077 & 0.767 & 0.531 & 1.532 \\
& 1.582 & 0.052 & 1.137 & 0.210 & 1.072 & 0.112 & 0.158 & 0.893 \\
\hline
\end{tabular}




\section{REFERENCES}

Alfarano, S., Lux, T. and Wagner F. (2005), 'Estimation of agent-based models: the case of an asymmetric herding model', Computational Economics 26, 19-49.

Aoki, M. and Yoshikawa, H. (2002), 'Demand saturation/creation and economic growth', Journal of Economic Behavior and Organization 48, 127-154.

Arthur, W., Holland, J., LeBaron, B., Palmer, R. and Tayler, P. (1997), 'Asset pricing under endogeneous expectations in an artifical stock market', Economic Notes 26(2), 297-330.

Baillie, R. T., Bollerslev, T. and Mikkelsen, H. (1996), 'Fractionally integrated generalized autoregressive conditional heteroskedasticity', Journal of Econometrics 74, 3-30.

Bollerslev, T. (1986), 'Generalized autoregressive conditional heteroskedasticity', Journal of Econometrics 31, 307-327.

Brock, W. and Hommes, C. (1997), 'A rational route to randomness', Econometrica 65, 1059-1095.

Brock, W. and Hommes, C. (1998), 'Heterogeneous beliefs and routes to chaos in a simple asset pricing model', Journal of Economic Dynamics and Control 22, 1235-1274.

Brock, W. and LeBaron, B. (1996), 'A structural model for stock return volatility and trading volume', Review of Economics and Statistics 78, 94-110.

Bullard, J. and Duffy, J. (1999), 'Using Genetic Algorithms to Model the Evolution of Heterogeneous Beliefs', Computational Economics 13, 41-60.

Chen, S.-H., Lux, T. and Marchesi, M. (2001), 'Testing for non-linear structure in an artificial financial market', Journal of Economic Behavior and Organization 46, 327-342.

Chen, S.-H. and Yeh, C.-H. (1997), 'Toward a computable approach to the efficient market hypothesis: an application of genetic programming', Journal of Economic Dynamics and Control 21, 1043-1063.

Chen, S.-H. and Yeh, C.-H. (2002), 'On the emergent properties of artificial stock markets: the efficient market hypothesis and the rational expectations hypothesis', Journal of Economic Behavior and Organization 49, 217-239.

Chiarella, C. (1992), 'The dynamics of speculative behavior', Annals of Operations Research 37, 101-123.

Chiarella, C., Dieci, R. and Gardini, L. (2002), 'Speculative behavior and complex asset price dynamics', Journal of Economic Behavior and Organization 49, 173-197.

Chiarella, C., Dieci, R. and Gardini, L. (2005), 'The dynamic interaction of spectulation and diversification', Applied Mathematical Finance 12, 17-52.

Chiarella, C., R. Dieci and X. He (2006a), 'Heterogeneous expectations and speculative behaviour in a dynamic multi-asset framework', Journal of Economic Behavior and Organization, in press.

Chiarella, C. and He, X. (2001), 'Asset pricing and wealth dynamics under heterogeneous expectations', Quantitative Finance 1, 509-526.

Chiarella, C. and He, X. (2002), 'Heterogeneous beliefs, risk and learning in a simple asset pricing model', Computational Economics 19, 95-132.

Chiarella, C. and He, X. (2003), 'Heterogeneous beliefs, risk and learning in a simple asset pricing model with a market maker', Macroeconomic Dynamics 7, 503-536.

Chiarella, C., He, X., Hung, H. and Zhu, P. (2006b), 'An analysis of the cobweb model with boundedly rational heterogeneous producers', Journal of Economic Behavior and Organization, in press.

Chiarella, C., He, X. and Wang, D. (2006c), 'A behavioral asset pricing model with time-varying second moment', Chaos, Solitons and Fractals 29, 535-555.

Chung, C.-F. (1999), 'Estimating the fractionally integrated GARCH model', discussion paper, National Taiwan University.

Dacorogna, M., Muller, U., Jost, C., Pictet, O., Olsen, R. and Ward, J. (1995), 'Heterogeneous real-time trading strategies in the foreign exchange market', European Journal of Finance 1, 383-403.

Day, R. and Huang, W. (1990), 'Bulls, bears and market sheep', Journal of Economic Behavior and Organization 14, 299-329.

DeGrauwe, P. and Grimaldi, M. (2003), 'Exchange rate puzzles: a tale of switching attractor', manuscript, University of Leuven.

DeLong, J., Shleifer, A., Summers, L. and Waldmann, R. (1990), 'Noise trader risk in financial markets', Journal of Political Economy 98, 703-738.

Dieci, R., I. Foroni, L. Gardini and X. He (2006), 'Market model, adaptive beliefs and asset price dynamics', Chaos, Solitons and Fractals 29 520-534.

Ding, Z., Granger, C. and Engle, R. (1993), 'A long memory property of stock market returns and a new model', Journal of Empirical Finance 1, 83-106.

Engle, R. (1982), 'Autoregressive conditional heteroscedasticity with estimates of the variance of UK inflation', Econometrica 50, 987-1008. 
Farmer, D. and Joshi, S. (2002), 'The price dynamics of common trading strategies', Journal of Economic Behavior and Organization 49, 149-171.

Frankel, F. and Froot, K. (1987), 'Using survey data to test propositions regarding exchange rate expectations', American Economic Review 77, 133-153.

Gaunersdorfer, A. (2000), 'Endogenous fluctuations in a simple asset pricing model with heterogeneous agents', Journal of Economic Dynamics and Control 24, 799-831.

Gaunersdorfer, A. and Hommes, C. (2006), 'A nonlinear structural model for volatility clustering', In: G. Teyssire and A. Kirman (eds.), Long Memory in Economics, Springer-Verlag, forthcoming.

Geweke, J. (1998), 'Comment on real and spurious long memory properties of stock-market data (by Lobato, I., and N. Savin)', Journal of Business \& Economic Statistics 16, 269-271.

Geweke, J. and Poter-Hudak, S. (1983), 'The estimation and application of long memory time series models', Journal of Time Series Analysis, 4, 221-238.

Giardina, I. and J.-P. Bouchaud. (2003), 'Bubbles, crashes and intermittency in agent based market models', European Physical Journal B, 31, 421-437.

He, X. and Li, Y. (2005), 'Heterogeneity, profitability, and autocorrelations', Research Paper 147, Quantitative Finance Research Centre, University of Technology, Sydney. Avilable at: www.business.uts.edu.au/qfrc/research/publications.html.

Hommes, C. (2001), 'Financial markets as nonlinear adaptive evolutionary systems', Quantitative Finance 1, 149_ 167.

Hommes, C. (2002), 'Modeling the stylized facts in finance through simple nonlinear adaptive systems', Proceedings of National Academy of Science of the United States of America 99, 7221-7228.

Iori, G. (2002), 'A microsimulation of traders activity in the stock market: the role of heterogeneity, agents' interactions and trade frictions', Journal of Economic Behavior and Organization 49, 269-285.

Kesten, H. (1973), 'Random difference equations and renewal theory for products of random matrices', Acta Mathematica 131, 207-248.

Kirman, A. (1991), Epidemics of opinion and speculative bubbles in financial markets, chapter 17 in Money and Financial Markets, M. Taylor Ed. pp. 354-368, Macmillan, London.

Kirman, A. (1993), 'Ants, rationality, and recruitment', Quarterly Journal of Economics 108, 137-156.

LeBaron, B. (2000), 'Agent based computational finance: suggested readings and early research', Journal of Economic Dynamics and Control 24, 679-702.

LeBaron, B. (2001), 'A builder's guide to agent-based financial markets', Quantitative Finance 1(2), $254-261$.

LeBaron, B. (2002), 'Calibrating an agent-based financial market to macroeconomic time series', Technical report, Brandeis University, Waltham, MA.

LeBaron, B., Arthur, W. and Palmer, R. (1999), 'Time series properties of an artifical stock market', Journal of Economic Dynamics and Control 23, 1487-1516.

Lewwllen, J. and Shanken, J. (2002), 'Learning, asset-pricing tests, and market efficiency', Journal of Finance 57(3), 1113-1145.

Li, Y., Donkers, B. and Melenberg, B. (2006a), 'The econometric analysis of microscopic simulation models', CentER Discussion Paper No. 2006-99, Tilburg University. Available at SSRN: http://ssrn.com/abstract=939518.

Li, Y., Donkers, B. and Melenberg, B. (2006b), 'The non- and semiparametric analysis of MS models: Some applications', CentER Discussion Paper No. 2006-95, Tilburg University. Available at SSRN: http://ssrn.com/abstract=939510.

Lux, T. (1995), 'Herd behavior, bubbles and crashes', Economic Journal 105, 881-896.

Lux, T. (1997), 'Time variation of second moments from a noise trader/infection model', Journal of Economic Dynamics and Control 22, 1-38.

Lux, T. (1998), 'The socio-economic dynamics of speculative markets: Interacting agents, chaos, and the fat tails of return distributions', Journal of Economic Behavior and Organization 33, 143-165.

Lux, T. (2004), 'Financial power laws: Empirical evidence, models, and mechanisms', in C. Ciotti ed., Power Law in the Social Sciences: Discovering Complexity and Non-Equilibrium in the Social Universe, submitted to Cambridge University.

Lux, T. and Marchesi, M. (1999), 'Scaling and criticality in a stochastic multi-agent model of a financial markets', Nature 397(11), 498-500.

Lux, T. and Marchesi, M. (2001), 'Volatility clustering in financial markets: A micro-simulation of interacting agents', Journal of Theoretical and Applied Finance 3, 675-702.

Lux, T. and Sornette, D. (2002), 'On Rational Bubbles and Fat Tails', Journal of Money, Credit and Banking 34, 589-610.

Lux, T. and S. Schornstein (2005), 'Genetic learning as an explanation of stylized facts of foreign exchange markets', Journal of Mathematical Economics 41, 169-196. 
Manzan, S. and Westerhoff, F. (2005), 'Representativeness of news and exchange rate dynamics', Journal of Economic Dynamics and Control 29, 677-689.

Pagan, A. (1996), 'The econometrics of financial markets', Journal of Empirical Finance 3, $15-102$.

Robinson, P. M. (1995), 'Log-periodgram regression of time series with long range dependence', Annals of Statistics 23, 1048-1072.

Robinson, P. M. and Henry, M. (1999), 'Long and short memory conditional heteroscedasticity in estimating the memory parameters of levels', Econometric Theory 15, 229-266.

Velasco C. (1999), 'Non-stationary log-periodgram regression', Journal of Econometrics 91, 325-371.

Westerhoff, F. (2003), 'Speculative markets and the effectiveness of price limits', Journal of Economic Dynamics and Control 28, 439-508.

Westerhoff, F. (2004), 'Multiasset market dynamics', Macroeconomic Dynamics 8, 596-616.

Westerhoff, F. and Dieci, R. (2006), 'The effectiveness of Keynes-Tobin transaction taxes when heterogeneous agents can trade in different markets: A behavioral finance approach', Journal of Economic Dynamics and Control 30, 293-322. 